\title{
A comparison of the endotoxin biosynthesis and protein oxidation pathways in the biogenesis of the outer membrane of Escherichia coli and Neisseria meningitidis
}

\author{
Susannah Piek and Charlene M. Kahler*
}

Department of Pathology and Laboratory Medicine, The University of Western Australia, Perth, WA, Australia

Edited by:

Martin J. McGavin, University of

Western Ontario, Canada

\section{Reviewed by:}

Jon Audia, University of South

Alabama School of Medicine, USA

Hui Wu, University of Alabama at

Birmingham, USA

*Correspondence:

Charlene M. Kahler, Department of Pathology and Laboratory

Medicine, The University fo Western Australia, Rm 2.03A, L Block, QEII

Medical Centre, Monash Avenue,

Crawley, Perth WA 6009, Australia.

e-mail: charlene.kahler@uwa.edu.au
The Gram-negative bacterial cell envelope consists of an inner membrane (IM) that surrounds the cytoplasm and an asymmetrical outer-membrane (OM) that forms a protective barrier to the external environment. The OM consists of lipopolysaccahride (LPS), phospholipids, outer membrane proteins (OMPs), and lipoproteins. Oxidative protein folding mediated by periplasmic oxidoreductases is required for the biogenesis of the protein components, mainly constituents of virulence determinants such as pili, flagella, and toxins, of the Gram-negative OM. Recently, periplasmic oxidoreductases have been implicated in LPS biogenesis of Escherichia coli and Neisseria meningitidis. Differences in OM biogenesis, in particular the transport pathways for endotoxin to the $\mathrm{OM}$, the composition and role of the protein oxidation, and isomerization pathways and the regulatory networks that control them have been found in these two Gram-negative species suggesting that although form and function of the OM is conserved, the pathways required for the biosynthesis of the $\mathrm{OM}$ and the regulatory circuits that control them have evolved to suit the lifestyle of each organism.

\footnotetext{
Keywords: oxidoreductases, disulfide bonds, protein oxidation, protein isomerization, lipopolysaccharides (LPS), lipooligsaccharides (LOS), Neisseria meningitidis
}

\section{THE GRAM-NEGATIVE CELL ENVELOPE}

The Gram-negative bacterial cell envelope consists of an inner membrane (IM) that surrounds the cytoplasm and an asymmetrical outer-membrane $(\mathrm{OM})$ that forms a protective barrier to the external environment (reviewed in Ruiz et al., 2006). The two membranes are separated by an aqueous compartment or periplasm which contains a thin peptidoglycan layer that contributes to cell structure and resistance to osmotic stress. Unlike the cytoplasmic compartment, the periplasm is an oxidizing environment that is devoid of ATP and comprises $10 \%$ of the cell volume. The Gram-negative OM coordinates interactions with the external environment, preventing the entry of toxic molecules while still allowing intake of nutrients and excretion of toxic waste products. The asymmetric OM consists of an inner leaflet composed of phospholipids and an outer leaflet composed of lipopolysaccahride (LPS), which is responsible for the highly efficient barrier function of the OM (Raetz and Whitfield, 2002). There are two types of proteins incorporated in the OM; outer membrane proteins (OMPs) and lipoproteins, which usually face the periplasm and form complexes with OMPs and periplasmic machinery. OMPs known as porins selectively allow intake of nutrients and expel toxic waste. OMPs can also have enzymatic functions and act as adhesins. As a result, the LPS and protein components of the OM have important roles in the survival and virulence of Gram-negative bacteria.

Oxidative protein folding mediated by periplasmic oxidoreductases is required for the biogenesis of the protein components, mainly constituents of virulence determinants such as pili, flagella, secretion systems and toxins, of the Gram-negative OM (Kadokura and Beckwith, 2010). Oxidoreductases, known as Disulfide bond (Dsb) proteins, catalyze disulfide bond formation in membrane and secreted proteins as they transit through the periplasm to the OM. Disulfide bonds are covalent linkages formed between thiol groups of two Cys residues and are necessary for the stability and/or activity of proteins. Recently, periplasmic oxidoreductases have been implicated in LPS biogenesis of Escherichia coli (Denoncin et al., 2010) and Neisseira meningitidis (Piek et al., 2012), suggesting a much larger role of these enzymes in OM biogenesis.

The structure and composition of the OM is conserved in all Gram-negative bacteria, regardless of the ecological niche of the organism. Comparative genomic studies have shown that epidemic pathogenic bacteria have reduced genomes both in size and regulatory complexity when compared to organisms that have a commensal lifestyle (Merhej et al., 2009; Georgiades, 2012). Pathogenic bacteria also acquire many virulence determinants which enable specialized niche adaptation. A recent study by Liechti and Goldberg (2012) has shown that genome reduction and niche specialization in $H$. pylori has resulted in a smaller number of components required for OMP, LPS, and lipoprotein transport pathways. In this review, the periplasmic protein folding and LPS biogenesis pathways of the extensively studied commensal E. coli, and epidemic pathogen, Neisseria meningitidis, are compared. Differences in OM biogenesis, in particular the transport pathways for endotoxin to the OM, the composition and role of the protein oxidation and isomerization 
pathways for protein folding in the periplasm and the regulatory networks that control them are elucidated. It appears that although form and function of the OM is conserved, the pathways required for the biosynthesis of the OM and the regulatory circuits that control them have evolved to suit the lifestyle of each organism.

\section{THE GRAM-NEGATIVE CELL ENVELOPE OF E. coli PERIPLASMIC OXIDOREDUCTASES AND THEIR ROLE IN OM BIOGENESIS IN E. coli}

Periplasmic protein folding catalyzed by Dsb proteins is best characterized in E. coli K12. Dsb proteins ensure correct disulfide bond formation through two independent but parallel pathways of periplasmic protein folding known as the oxidation and isomerization pathways. The oxidation pathway (DsbA/DsbB system) is primarily responsible for the catalysis of disulfide bond formation between adjacent Cys residues of protein substrates while the isomerization pathway (DsbD/DsbC system) re-shuffles any incorrectly formed disulfide bonds, which is important if the native protein contains multiple disulfide bonds between non-consecutive Cys residues (Figure 1).

\section{Oxidation pathway of periplasmic protein folding in E. coli}

The oxidation pathway is required for the correct folding of a number of virulence proteins of $E$. coli. In fact, there are approximately 300 disulfide-containing proteins that transit through the periplasm of E. coli (Hiniker and Bardwell, 2004; Dutton et al., 2008) and are therefore substrates of the oxidation pathway

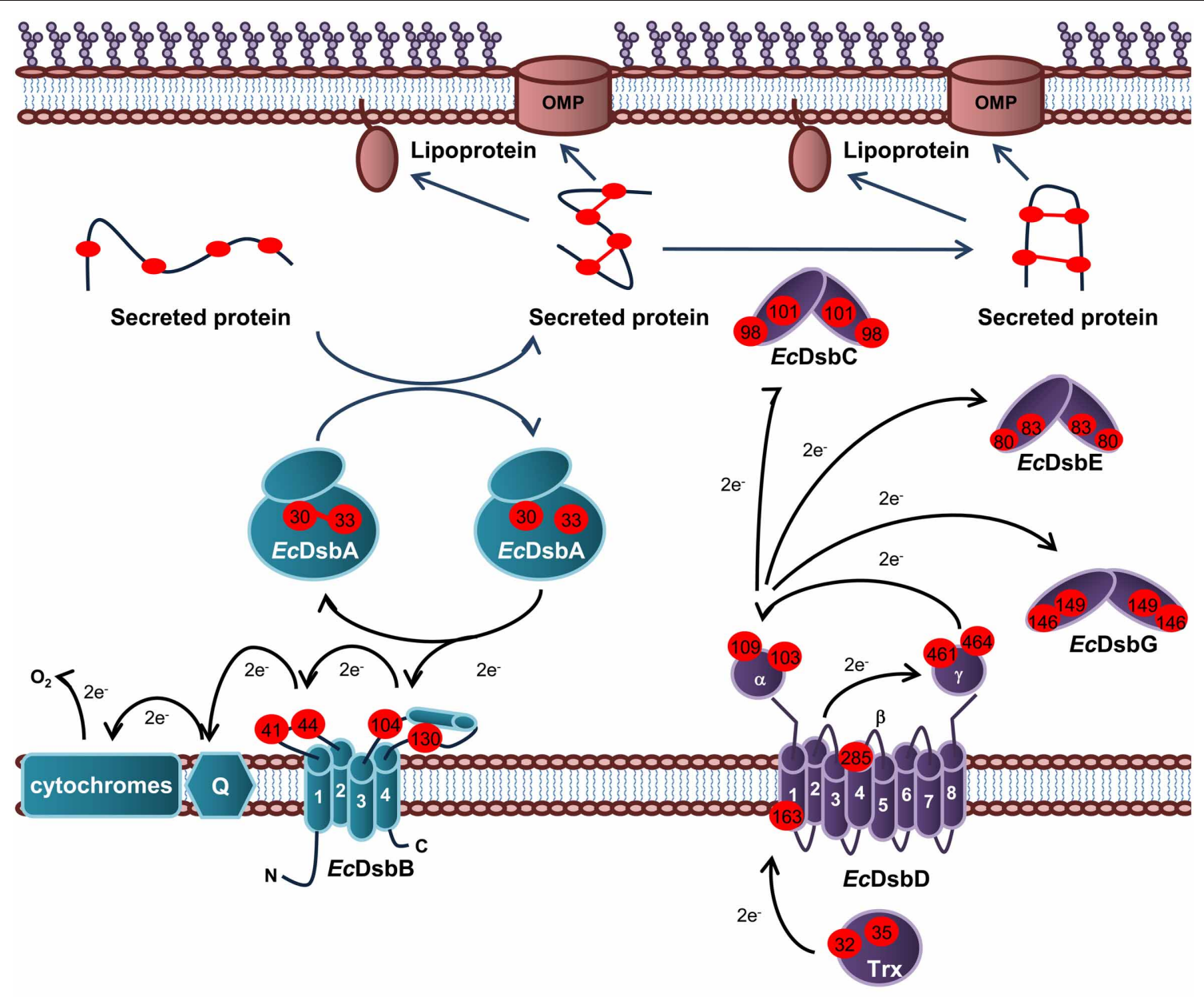

Oxidation

FIGURE 1 | Oxidation and isomerization pathways of periplasmic protein folding in $\boldsymbol{E}$. coli. Oxidation pathway: The soluble oxidoreductase EcDsbA catalyses disulfide bond formation between adjacent cysteine residues of secreted proteins. EcDsbA is re-oxidized by the $I M$ bound $E c D s b B$. Under aerobic growth conditions, electrons flow from $E c D s b B$ to molecular oxygen via ubiquinone (Q) and cytochrome. Isomerization pathway: The oxidoreductase $E c D s b C$ re-shuffles any incorrect disulfide bonds which is important in secreted proteins that

\section{Isomerisation}

contain multiple disulphide bonds between non-consecutive cysteine residues. $E_{C D s b C}, E_{C D s b G}$, and $E_{C} \mathrm{DsbE}$ are kept in a reduced state by the $I M$ bound $E c D s b D$ which in turn is reduced by passing electrons to thioredoxin in the cytoplasm. The oxidation pathway is in turquoise and the isomerization pathway is in purple. Cysteine residues are denoted by a red circle containing the residue number. The direction of electron transfer is shown by black arrows while protein folding is shown with dark blue arrows. 
of periplasmic protein folding. Central to this pathway is the oxidoreductase $E c D s b A$; the primary disulfide donor that interacts with these substrates (Bardwell et al., 1991). EcDsbA is kept in an oxidized and active state by the membrane bound EcDsbB (Bardwell et al., 1993), which in turn is re-oxidized by passing electrons via quinones $(\mathrm{Q})$ to the electron transport pathway in the cytoplasmic membrane (Bader et al., 1999; Kobayashi and Ito, 1999). There are a number of features of both EcDsbA and $E c \mathrm{DsbB}$ that drive the flow of electrons from the reduced substrate through to the electron transport pathway.

$E c D s b A$ is a monomeric $21 \mathrm{kDa}$ periplasmic enzyme that belongs to the thioredoxin superfamily (Martin et al., 1993a). Features common to this superfamily include an active site motif consisting of two Cys residues separated by two amino acids (CXXC) embedded in a thioredoxin-like fold (Martin, 1995). $E c \mathrm{DsbA}$ differs from other members of the thioredoxin superfamily in that it contains an extra alpha $(\alpha)$-domain (65 residues forming four $\alpha$-helices) inserted into the center of the thioredoxin domain. The $\alpha$-domain forms a globular-like cap over the active site $\left(\mathrm{C}_{30} \mathrm{PHC}_{33}\right)$ located at the $\mathrm{N}$-terminus of the first $\alpha$-helix of the thioredoxin domain (Martin et al., 1993a). Oxidized EcDsbA contains a disulfide bond between the two Cys residues of the active site which is donated to an unfolded protein substrate during protein oxidation. EcDsbA-substrate disulfide exchange proceeds as a biomolecular nucleophilic substitution reaction.

$E c D s b A$ is an extremely efficient oxidizing disulfide catalyst that rapidly oxidizes substrate proteins. With a standard redox potential of $\sim-120 \mathrm{mV}, E c \mathrm{DsbA}$ is one of the strongest thiol oxidants (Huber-Wunderlich and Glockshuber, 1998), which can be explained in part by biophysical properties. The active site $\mathrm{C}_{30}$ is surface exposed and has an unusually low $\mathrm{p} K_{\mathrm{a}}$ of $\sim 3.3$ (the normal $\mathrm{p} K_{\mathrm{a}}$ of Cys is 9) (Nelson and Creighton, 1994; HuberWunderlich and Glockshuber, 1998). As $\mathrm{C}_{30}$ is a thiolate anion at physiological $\mathrm{pH}$, the oxidized form of EcDsbA is less stable than the reduced form and is therefore more reactive. This drives the thermodynamic flow of electrons from the substrate to EcDsbA and the transfer of the disulfide from the $E c D s b A$ active site to the substrate.

Another important structural feature of EcDsbA is the loop between $\alpha 6$ and $\beta 4$, which contains the highly conserved cisPro $_{151}$ residue that impacts directly on the active site motif and forms the other half of the active site in most members of the thioredoxin superfamily (Martin et al., 1993b; Martin, 1995). Mutating the cis-Pro 151 to a Thr resulted in accumulation of mixed-disulfide intermediate complexes, suggesting a role of $c i s-P_{151}$ in EcDsbA substrate release (Kadokura et al., 2004). The residue immediately preceding cis-Pro 151 in $\mathrm{EcDs}_{15}$ $\left(\mathrm{V}_{150}\right)$ modulates redox potential and coordinates interactions with $E c D s b B$ and substrate proteins. In addition, the solved structure of $E c D s b A$ in a complex with a peptide substrate revealed that the residues of the loop between $\alpha 6$ and $\beta 4, V_{145} Q_{L R G V}$, as well as the residues of the type IV $\beta$ turn between $\beta 3$ and $\alpha 2, \mathrm{~F}_{63} \mathrm{MGG}_{66}$, form the substrate binding surface of EcDsbA (Paxman et al., 2009). Interestingly, these residues have also been implicated in interaction with $E c \mathrm{DsbB}$.

$E c D s b B(20 \mathrm{kDa})$ consists of four trans-membrane $\alpha$ helices connected by two periplasmic loops that contain two intra-molecular disulfide bonds, between $\mathrm{C}_{41}$ and $\mathrm{C}_{44}$ in the first periplasmic loop, and $\mathrm{C}_{104}$ and $\mathrm{C}_{130}$ in the second periplasmic loop, that are essential to EcDsbA oxidation (Jander et al., 1994; Kadokura and Beckwith, 2002). Co-crystallization of the EcDsbA (C33A) in a covalently linked complex with EcDsbB (C130S) revealed the residues $\mathrm{P}_{100}$ FATCDF $_{106}$ of the second periplasmic loop of $E c \mathrm{DsbB}$ is accommodated in the hydrophobic groove of EcDsbA (Inaba et al., 2006a). This structure also revealed that the $E c$ DsbB residues $\mathrm{C}_{104} \mathrm{DF}_{106}$ interact with the residues $\mathrm{R}_{148} \mathrm{GV}_{150}$ of $E c \mathrm{DsbA}$ to form a short anti-parallel $\beta$-sheet, similar to the interaction between EcDsbA and substrates (Paxman et al., 2009). It appears that the relative specificity of reduced $E c D s b A$ for $E c \mathrm{DsbB}$ could be a result of the additional interactions of $E c \mathrm{DsbB}$ with residues located within the hydrophobic groove of EcDsbA. Recent NMR analysis of the EcDsbA homolog in Vibrio cholorae $(V c \mathrm{DsbA})$ revealed greater interdomain flexibility resulting in the widening of the hydrophobic groove in the reduced form of the enzyme (Horne et al., 2007). This suggests that the reduced form of EcDsbA could be in a more open conformation allowing $E c D s b B$ interaction with the hydrophobic groove.

The transfer of electrons from EcDsbA to $E c \mathrm{DsbB}$ is an energetically unfavorable reaction as the standard redox potential values for the $E c$ DsbB Cys pairs $\left(-210 \mathrm{mV}\right.$ for $\mathrm{C}_{41}-\mathrm{C}_{44}$ and $-220 \mathrm{mV}$ for $\mathrm{C}_{104}-\mathrm{C}_{130}$ ) (Inaba et al., 2005) are much lower than that of $E c$ DsbA $\left(-122 \mathrm{mV}\right.$ for $\mathrm{C}_{30}-\mathrm{C}_{33}$ ) (Wunderlich et al., 1993). To overcome this barrier, disulfide exchange is initiated by interaction of the second periplasmic loop of $E c D s b B$ with the open hydrophobic groove of reduced $E c D s b A$. This interaction induces a conformational change in the second periplasmic loop of $E c \mathrm{DsbB}$ so that $\mathrm{C}_{104}$ and $\mathrm{C}_{130}$ are separated spatially, with $\mathrm{C}_{130}$ situated closer to the $\mathrm{C}_{41}-\mathrm{C}_{44}$ disulfide of $E c \mathrm{DsbB}$. It is thought that $E c D s b A$ induced separation of the $\mathrm{C}_{104}-\mathrm{C}_{130}$ disulfide of $E c D s b B$ is what makes transfer of the disulfide bond from $E c D s b B$ to EcDsbA an energetically favorable reaction (Kadokura and Beckwith, 2002; Tapley et al., 2007; Inaba et al., 2009). The solved EcDsbA (C33A) - EcDsbB (C130S) crystal structure revealed a possible role of $\mathrm{M}_{64}$ of $E c \mathrm{DsbA}$ in separation of the $\mathrm{C}_{104}-\mathrm{C}_{130}$ disulfide as it appeared to intervene between $\mathrm{C}_{104}$ and the serine at position 130 (Inaba et al., 2006a). Interestingly, this residue was also implicated in EcDsbA interactions with substrates (Paxman et al., 2009). Upon separation of the $\mathrm{C}_{104}$ and $\mathrm{C}_{130}$ residues of $E c \mathrm{DsbB}$, the thiolate anion $\mathrm{C}_{30}$ of reduced $E c \mathrm{DsbA}$ attacks $\mathrm{C}_{104}$ of $E c \mathrm{DsbB}$ forming a mixed disulfide bridge (Kadokura and Beckwith, 2002). This mixed disulfide is then resolved by one of two possible pathways. In the rapid pathway, oxidized EcDsbA is released prior to oxidation of $E c \mathrm{DsbB}$ and in the slow pathway, oxidized EcDsbA is released simultaneously with oxidized EcDsbB (Inaba and Ito, 2008). In both pathways, $\mathrm{C}_{44}$ of EcDsbB is transiently reduced, which leads to the formation of a charge transfer complex between $E c$ DsbB and ubiquinone (UQ) (Inaba et al., 2006a; Zhou et al., 2008).

It has been proposed that under aerobic conditions, oxidation of the $\mathrm{C}_{41}-\mathrm{C}_{44}$ residues of $E c \mathrm{DsbB}$ is initiated by transfer of a partial charge to the benozoquinone ring of UQ by the thiolate anion $\mathrm{C}_{44}$ of reduced $E c \mathrm{DsbB}$. This is followed by $\mathrm{C}_{44}$ nucleophilic attack of UQ to form a charge-transfer complex between $\mathrm{UQ}$ and $E c \mathrm{DsbB}$ that is stabilized by $\mathrm{R}_{48}$ of $E c \mathrm{DsbB}$ (Kadokura 
et al., 2000; Kobayashi et al., 2001; Inaba et al., 2006b). The existence of a charge transfer complex is supported by quantum chemical simulation by Inaba et al. (2006b), and the observation that $E c D s b B$ elicits a characteristic red shift of bound UQ during the EcDsbA oxidation (Inaba et al., 2004, 2006b). The $E c D s b A(C 33 A)-E c D s b B(C 130 S)$ crystal structure supports this model for sequential disulfide exchange as the six redox-active Cys residues are arranged in a straight line. This structure also shows $\mathrm{C}_{44}$ of $E c \mathrm{DsbB}$ is well positioned to form a charge transfer complex with UQ (Inaba et al., 2006a).

\section{Alternatives to the classical oxidation pathway of periplasmic protein folding in E. coli}

The classic protein oxidation pathway represented by $E c D s b A$ and $E c D s b B$ has been shown to be responsible for introducing disulfide bonds into the vast majority of proteins in E. coli (Bardwell et al., 1991). However, exceptions to this classic or generalist pathway have increasingly been found and are characterized by oxidoreductases which are specialized for the recognition of a specific or a small array of substrates. Pathways which contain specialized oxidoreductases fall into two separate categories. The first category consists of specialist oxidoreductases, which are partnered with a specific redox partner. As an example, uropathogenic E. coli (UPEC) and Salmonella enterica serovar Typhimurium contain a second functional redox pair, DsbL and DsbI, which are $19 \%$ and $24 \%$ identical in sequence to EcDsbA and $E c D s b B$, respectively. DsbL specifically oxidizes the substrate arylsulfate sulfotransferase (ASST), for which there is no virulence phenotype (Grimshaw et al., 2008; Lin et al., 2009). The genes encoding DsbL, DsbI, and ASST are organized in a tricistronic operon that is found throughout Salmonella and in a subset of Enterobacteriacea (Grimshaw et al., 2008). Interestingly, DsbL and DsbI act as a specific redox pair that is independent and does not interact with the classic pathway (in other words, DsbL cannot ultilize $E c \mathrm{DsbB}$ as a redox partner).

The second category of specialist oxidoreductase does not have a specialist redox partner but have a reduced substrate repertoire. In this example, S. enterica serovar Typhimurium contains a virulence plasmid encoding a third DsbA-like protein, SeSrgA (SdiA-regulated gene) (Bouwman et al., 2003), in addition to the chromosomally encoded specialist oxidoreductase, SeDsbL, and the generalist oxidoreductase, SeDsbA. SeSrgA has a narrow substrate range as it only efficiently oxidizes two substrates, PefA (plasmid encoded fimbrae) (Bouwman et al., 2003), and SpiA, a component of the Type III secretion system (Miki et al., 2004). Interestingly, SeSrgA does not have its own redox partner but is dependent on $\mathrm{SeDsbB}$ of the classic oxidation pathway (Bouwman et al., 2003). SeDsbL and SeSrgA contain the active site residues of $\mathrm{CPFC}$ and $\mathrm{CPPC}$, respectively, corresponding to $\mathrm{C}_{31} \mathrm{PHC}_{33}$ in $E c \mathrm{DsbA}$, and have very different redox potentials of $\sim-95 \mathrm{mV}$ and $\sim-154 \mathrm{mV}$, respectively, compared to $\sim-120 \mathrm{mV}$ of $E c \mathrm{DsbA}$.

\section{Isomerization pathway of periplasmic protein folding in $E$. coli}

The isomerization ( $E c \mathrm{DsbC} / E c \mathrm{DsbD})$ pathway is responsible for the re-shuffling of incorrect disulfide bonds introduced by EcDsbA into the periplasmic proteins of E. coli
(Rietsch et al., 1996). Incorrect disulfide bond formation may occur if the bonds need to form between non-consecutive Cys residues (Berkmen et al., 2005). As membrane and secreted proteins of prokaryotes rarely contain multiple disulfides (Hiniker and Bardwell, 2004), the formation of a single disulfide bridge between two adjacent Cys residues is often sufficient for correct protein folding. As a result, the oxidation pathway plays a much larger role in periplasmic protein folding of E. coli than the isomerization pathway (Yu and Kroll, 1999).

$E c \mathrm{DsbC}$ is the major protein disulfide isomerase central to the isomerization pathway and is kept in the reduced "active form" by the IM membrane bound EcDsbD which in turn is reduced by thioredoxin/thioredoxin reductase and NAPDH (Joly and Swartz, 1997; Rietsch et al., 1997). EcDsbC is a V-shaped homodimer consisting of two $23 \mathrm{kDa}$ monomers. The monomers consist of a C-terminal thioredoxin domain and an $\mathrm{N}$-terminal dimerization domain connected by a linker sequence (McCarthy et al., 2000). The active site residues $\mathrm{C}_{98} \mathrm{GYC}_{101}$ [redox potential of $-130 \mathrm{mV}$ (Zapun et al., 1995)] are located in the thioredoxin domain and are positioned so as to face the inside of the $\mathrm{V}$-shaped homodimeric structure (McCarthy et al., 2000). This surface is occupied primarily by hydrophobic residues which presumably aids interaction with a variety of substrates. In addition, $E c D s b C$ displays periplasmic chaperone activity that would facilitate substrate interactions (Chen et al., 1999). While dimerization of the $\mathrm{EcDsbC}$ monomers does not appear to be necessary for isomerase activity of the enzyme, it is important for protection of the active site Cys residues from oxidation by EcDsbB (Bader et al., 2001). Therefore, it has been suggested that the dimeric structure of $E c$ DsbC evolved to limit cross talk between the oxidation and isomerization pathways of periplasmic protein folding.

There are two models for EcDsbC-mediated protein disulfide isomerization both of which begin with nucleophilic attack of the incorrect disulfide bond in the substrate by $\mathrm{C}_{98}$ of reduced $E c D s b C$ resulting in the formation of an $E c D s b C$-substrate mixed disulfide intermediate complex. The intermediate complex is resolved by either attack of the mixed disulfide by a third Cys of the substrate resulting in correct disulfide bond formation in the substrate and oxidized EcDsbC or by attack of the disulfide by $\mathrm{C}_{101}$ of $E c \mathrm{DsbC}$ resulting in reduced substrate, that could then be correctly oxidized by EcDsbA (Rietsch et al., 1997; Walker and Gilbert, 1997; Darby et al., 1998). Either way, EcDsbC must be maintained in the reduced form to initiate disulfide bond isomerization.

In addition to $E c D s b C, E$. coli contains two substrate specific periplasmic disulfide isomerases, known as EcDsbG (Bessette et al., 1999) and EcDsbE (Reid et al., 2001). EcDsbC and EcDsbG share $24 \%$ amino acid identity, exhibit a conserved tertiary structure and are both maintained in a reduced state in the periplasm by the membrane bound $E c D s b D$ (Bessette et al., 1999; Reid et al., 2001). However, the V-shaped cleft is much wider in EcDsbG and more acidic when compared to that of EcDsbC (Heras et al., 2004). It was recently shown that EcDsbG preferentially interacts with periplasmic transpeptidases that cross link the major OM lipoprotein to peptidoglycan of E. coli (Depuydt et al., 2009). These enzymes contain a single Cys that is essential for enzymatic activity and which when attacked by oxygen radicals, will form 
a sulfenic acid adduct that inactivates the enzyme. It therefore appears that $E c D s b G$ plays a role in protecting proteins with a single Cys residue from sulfenylation in the periplasm. The large, hydrophilic binding cleft of EcDsbG could result in preferential binding of this isomerase to large folded proteins with a single oxidized Cys residue which cannot be accessed by $E c D s b C$. $E c D s b E$ (also known as $\mathrm{CcmG}$ ) is required for cytochrome $\mathrm{C}$ biogenesis (Reid et al., 2001). For simplicity this review will focus on $E c$ DsbD reduction of $E c$ DsbC.

The IM membrane bound oxidoreductase EcDsbD mediates trans-membrane electron transfer from cytoplasmic thioredoxin to periplasmic $E c \mathrm{DsbC}$ therefore maintaining $E c \mathrm{DsbC}$ in the reduced "active" form (Rietsch et al., 1997). EcDsbD consists of a periplasmic $\mathrm{N}$-terminal domain with an immunoglobulin-like fold $(E c \mathrm{DsbD} \alpha)$, a hydrophobic core $(E c \mathrm{DsbD} \beta)$ and periplasmic C-terminal domain $(E c \mathrm{DsbD} \gamma)$ with a thioredoxin-like fold (Haebel et al., 2002; Rozhkova et al., 2004; Cho et al., 2007). Each domain of EcDsbD contains two Cys residues; $\mathrm{C}_{103}$ and $\mathrm{C}_{109}$ in $E c \mathrm{DsbD} \alpha$ (redox potential $\sim-229 \mathrm{mV}$ ); $\mathrm{C}_{163}$ and $\mathrm{C}_{285}$ in $E c \mathrm{DsbD} \beta$ redox potential $\sim-246 \mathrm{mV}$ ); and $\mathrm{C}_{461}$ and $\mathrm{C}_{464}$ in EcDsbD $\gamma$ (redox potential $\sim-241 \mathrm{mV}$ ) (Rozhkova and Glockshuber, 2008). These Cys residues are essential for reduction of EcDsbC (Katzen and Beckwith, 2000).

$E c D s b D$ mediated reduction of $E c D s b C$ occurs through a disulfide bond cascade from $E c D s b C$ to thioredoxin in the cytoplasm (Katzen and Beckwith, 2000; Collet et al., 2002). EcDsbD $\beta$ consists of eight trans-membrane domains (TM1-TM8) with $\mathrm{C}_{163}$ and $\mathrm{C}_{285}$ present in the C-terminal regions of TM1 and TM4, respectively. Recent studies by Cho et al. (Cho et al., 2007; Cho and Beckwith, 2009) suggest $E c \operatorname{DsbD} \beta$ exhibits an hourglass-like structure with inverted symmetry in which the surfaces displaying $\mathrm{C}_{163}$ and $\mathrm{C}_{285}$ face the cytoplasm and periplasm, respectively. This arrangement appears to allow $\mathrm{C}_{163}-\mathrm{C}_{285}$ interaction with thioredoxin in the cytoplasm and $E c D s b D \gamma$ in the periplasm and therefore facilitate trans-membrane transfer of electrons (Katzen and Beckwith, 2000; Collet et al., 2002; Haebel et al., 2002; Rozhkova et al., 2004). In addition, examination of the redox potentials of each of the Cys pairs involved in reduction of EcDsbC reveals transfer of electrons from thioredoxin to $E c D s b C$ to be a thermodynamically favorable reaction (Rozhkova and Glockshuber, 2008).

\section{The role of periplasmic oxidoreductases in virulence of $E$. coli}

Periplasmic oxidoreductases are required for the biogenesis of the protein component of the OM of Gram-negative bacteria. As such, they are responsible for the correct folding of virulence proteins that are expressed on or around the OM (Figure 2). EcDsbA is central to the virulence of pathogenic E. coli as it is required for the biogenesis of machinery involved in adhesion to host cells, cellular spread, and secretion of effector molecules (Heras et al., 2009). Recently, the DsbA proteins of E. coli have been implicated in the biogenesis of the LPS component of the OM (Denoncin et al., 2010).

$E c \mathrm{DsbA}$ is required for the biogenesis of virulence factors involved in adhesion and movement of pathogenic E. coli. The biogenesis of the P fimbriae of uropathogenic E. coli (UPEC) is dependent on EcDsbA (Jacob-Dubuisson et al., 1994). Without functional P fimbriae, UPEC is unable to attach to urinary epithelial cells and is avirulent. $\mathrm{P}$ fimbriae consist of six subunit proteins that form a rod-like structure (Hultgren et al., 1991, 1993). The major P fimbrial rod subunit is PapA with PapG forming the adhesin while PapK, PapE, and PapF are the adaptor proteins. The PapC usher protein, which is integrated in the OM and acts as a scaffold protein, coordinates the polymerization of the fimbriae subunits. The periplasmic chaperone PapD binds to the individual subunits and targets them to the PapC usher protein in the OM (Hultgren et al., 1991, 1993). EcDsbA is required for the correct folding of the chaperone protein PapD and the adhesion protein PapG (Jacob-Dubuisson et al., 1994). In addition, the formation of the Type IV pili or bundle forming pili (BFP) of Enteropathogenic E. coli (EPEC) is dependent on EcDsbA. The BFP mediates adhesion and colonization of intestinal epithelial cells and a form of movement referred to as twitching motility (Pelicic, 2008). EcDsbA is required for the formation of a single intramolecular disulfide bond in $\mathrm{BfpA}$, the major subunit of BFP (Zhang and Donnenberg, 1996; Vogt et al., 2010). In the absence of EcDsbA, BfpA is rapidly degraded in the periplasm, resulting in an inability of EPEC to form functional BFP (Zhang and Donnenberg, 1996). Lastly, flagella are complex cell surface organelles that mediate bacterial motility. In E. coli, EcDsbA catalyzes the formation of a disulfide bond in FlgI, the flagellar P ring motor protein (Dailey and Berg, 1993). This critical disulfide is required for the biogenesis of functional flagella (Hizukuri et al., 2006). As a result, EcDsbA is required for the cellular spread of pathogenic E. coli and attachment to host cells.

The Type III secretion system is required for secretion of effector proteins into host cells (Cornelis, 2006; Galan and WolfWatz, 2006). The Type III secretion system of Gram-negative bacteria is comprised of more than 20 proteins. It consists of a basal body anchored in the IM, linked to a needle that extends from the bacterial surface. The secretin, that is integrated in the OM forms a major structural component of the Type III secretion system and consists of 12-14 subunits arranged in an oligomeric ring-like structure (Bitter, 2003). In $E$. coli, $E c D s b A$ is required for the correct folding of the secretin and therefore the biogenesis of the Type III secretion system (Miki et al., 2008). An example of an effector protein in E. coli is the heat labile enterotoxin from enterotoxigenic E. coli (ETEC), that results in the acute diarrhoea associated with an ETEC infection. The heat labile toxin is an $\mathrm{AB}_{5}$ toxin that contains a critical disulfide bond in the $\mathrm{B}$ monomer that is required for oligomerization of the subunits (Yu et al., 1992; Wulfing and Rappuoli, 1997).

Recently, the oxidoreductases $E c D s b A$ and $E c D s b C$ have been shown to be essential for the biogenesis of E. coli LPS. Specifically, the highly conserved and essential OMP, LptD, contains a critical disulfide bond that is required for its role in integration of newly synthesized LPS in the OM of E. coli (Denoncin et al., 2010). LPS constitutes $70 \%$ of the outer leaflet of the OM and plays a significant role in the virulence of $E$. coli. The establishment of this link between periplasmic oxidoreductases and LPS biogenesis suggests a much larger role for oxidoreductases in OM formation in E. coli. The processes involved in LPS biogenesis and the role of LPS in virulence of $E$. coli have been extensively studied. 


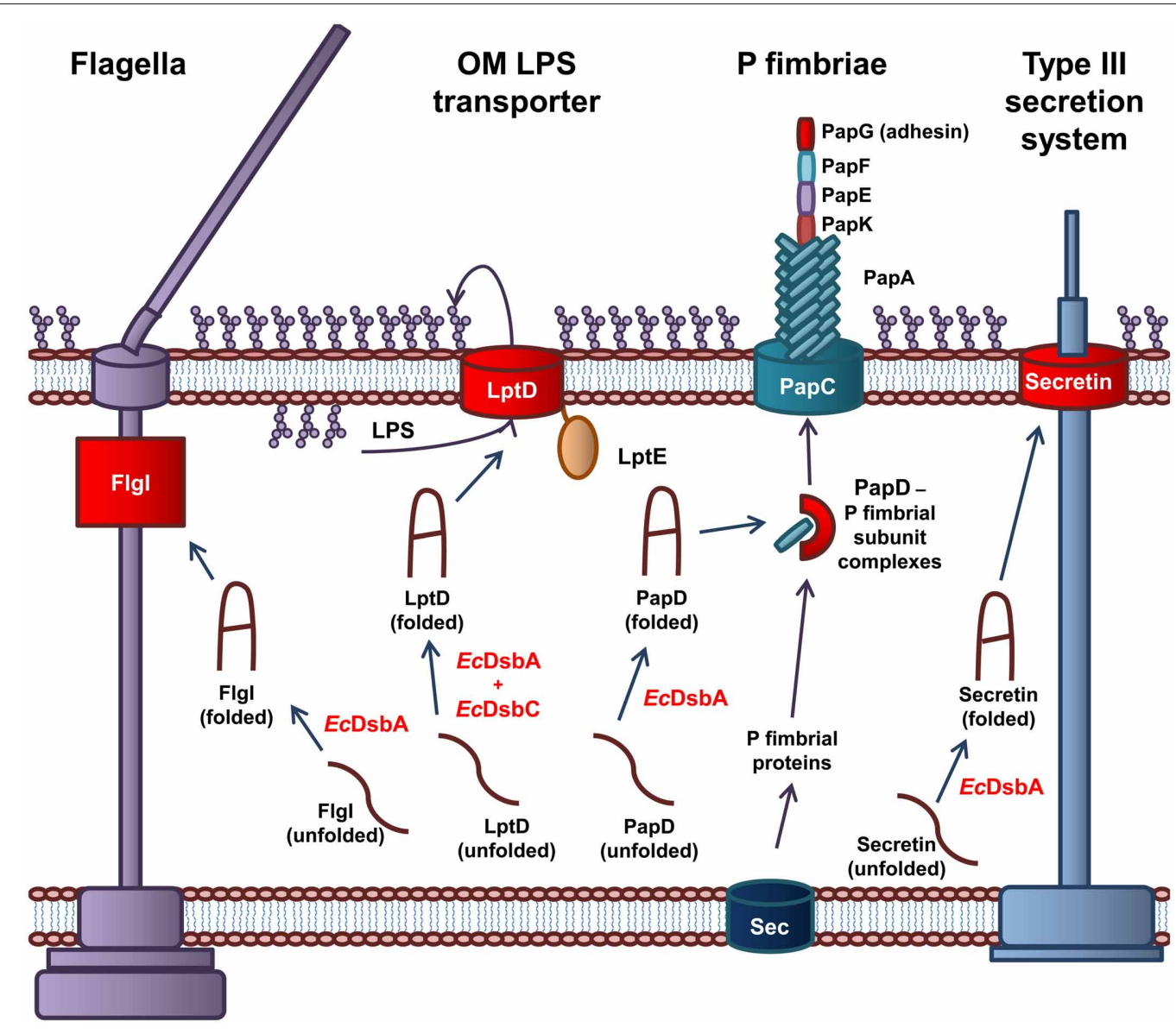

FIGURE 2 | The role of $E c$ DsbA and $E c$ DsbC in biosynthesis of virulence factors in $\boldsymbol{E}$. coli. EcDsbA catalyzes the formation of a disulfide bond in the Flgl P-ring motor protein required for assembly of functional flagella. Both $E c D s b A$ and $E c D s b C$ are required for the correct folding of the OMP LptD, which associates with the OM lipoprotein, LptE, to form the OM LPS transporter. EcDsbA catalyzes the formation of a disulfide bond in the PapD chaperone, which transports the $P$ fimbriae subunits from the Sec translocon in the IM to the usher protein $\mathrm{PapC}$ in the $\mathrm{OM}$, which coordinates the assembly of the P fimbraie. EcDsbA catalyses the formation of a disulfide bond in the OM secretin of the Type III secretion system. Protein folding reactions are shown by dark blue arrows, while cellular processes are shown by dark purple arrows.

\section{BIOSYNTHESIS AND TRANSPORT OF LPS TO THE OM IN E. coli AND ROLE IN PATHOGENESIS}

The outer leaflet of the Gram-negative OM is composed mainly of LPS which forms a highly protective barrier to the external environment. The structure of the archetypal model of LPS of E. coli consists of three distinct regions; lipid A which forms the hydrophobic membrane anchor, core oligosaccharide (OS) and repeating polysaccharide or $\mathrm{O}$-antigen (Raetz and Whitfield, 2002) (Figure 3). The lipid A is the toxic component of bacterial LPS (Galanos et al., 1985) while the $\mathrm{O}$ antigen provides a variable hydrophilic surface layer that can mask underlying antigenically-conserved core epitopes (Stenutz et al., 2006). The conserved OS core region of LPS consists of two L-glycero-Dmanno-heptose (Hep) and two 3-deoxy-D-manno-octulosonic acid (Kdo) residues attached to lipid A. Various sugars can be added to the $\mathrm{Hep}_{2}-\mathrm{Kdo}_{2}$-lipid A structure to complete the formation of the inner OS core (lipid A proximal) and outer OS core (attachment site for $\mathrm{O}$ antigen) regions of LPS. These additions are the basis of core typing in E. coli while the composition of the
$\mathrm{O}$ antigen attached to the outer core of LPS is the basis of antigenic variation.

\section{Biosynthesis of E. coli LPS}

Biosynthesis of E. coli LPS is initiated in the cytoplasm with the synthesis of $\mathrm{Kdo}_{2}$-lipid A region of LPS, which is conserved among Gram-negative bacteria and is the minimum structure required for growth in E. coli (Galloway and Raetz, 1990; Onishi et al., 1996). Construction of the $\mathrm{Kdo}_{2}$-lipid A region of LPS has been extensively studied in E. coli and is reviewed elsewhere (Raetz and Whitfield, 2002). This structure is then further modified to form the core OS-lipid A region before translocation across the IM upon which the $\mathrm{O}$-antigen is added and the complete LPS transported to the OM.

The conserved inner OS core-lipid A structure of E. coli LPS consists of $\mathrm{Hep}_{3}-\mathrm{Kdo}_{2}$-lipid A with the HepI and HepII residues phosphorylated at the $4^{\prime}$ positions (Raetz and Whitfield, 2002). To construct the $\mathrm{Hep}_{3}-\mathrm{Kdo}_{2}$-lipid A structure, the $\alpha 1,5$ heptosyltransferase, WaaC, transfers a heptose residue to $\mathrm{KdoI}$ of 


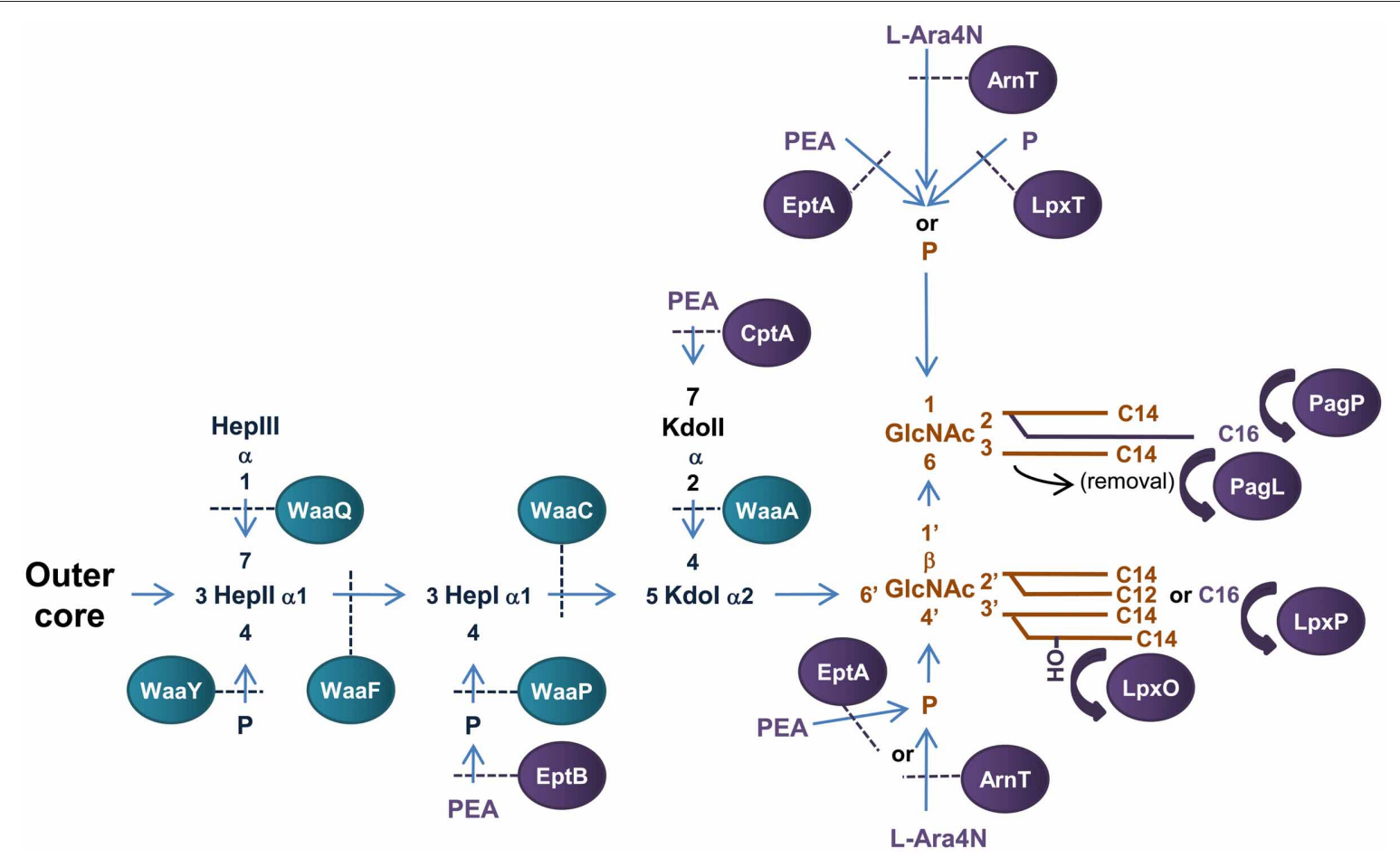

FIGURE 3 | Structure and biosynthesis of $\boldsymbol{E}$. coli LPS. The conserved Lipid A region is in orange while the conserved inner OS core region is in dark blue and variable modifications to these regions are in purple. Glycosyltransferases that form the inner OS core backbone are denoted by the blue boxes, and enzymes that modify the structure are in purple. The number of carbons present in each acyl chain is denoted by $C$ followed by a number. Structure and biosynthesis of the lipid A region is reviewed in detail elsewhere (Raetz and Whitfield, 2002). Refer to text for details on enzyme functions.
$\mathrm{Kdo}_{2}$-lipid A to form $\mathrm{Hep}_{1}-\mathrm{Kdo}_{2}$-lipid A (Gronow et al., 2000; Zamyatina et al., 2000). The $\alpha 1,3$ heptosyltransferase, WaaF, then catalyzes the transfer of the second heptose residue to HepI to form the basic conserved LPS inner core structure of $\mathrm{Hep}_{2}$ $\mathrm{Kdo}_{2}$-lipid A (Gronow et al., 2000; Zamyatina et al., 2000). The LPS kinase, WaaP, then phosphorylates the $4^{\prime}$ position of HepI which is necessary for any further modification of the core structure (Yethon et al., 1998; Yethon and Whitfield, 2001). In fact, mutants in waaP exhibit a deep rough phenotype and are avirulent (Yethon et al., 2000a,b). Upon phosphorylation of HepI by WaaP, the LPS kinase, WaaY catalyzes the transfer of phosphate to HepII. This is followed by the addition of a heptose residue to HepII by the $\alpha 1,7$ heptosyltransferase, WaaQ (Yethon et al., 1998). This step completes the formation of the conserved inner OS core-lipid A of E. coli LPS.

There are five known core types (R1, R2, R3, R4, and K-12) based on the variable regions of the inner and outer OS core regions of $E$. coli LPS, the structure and biogenesis of which has been reviewed elsewhere (Raetz and Whitfield, 2002). An interesting example of a variable addition to the inner OS core region is the addition of a third Kdo residue to the $4^{\prime}$ position of KdoII of the inner OS core of $E$. coli core types R 2 and K-12 catalyzed by the $\alpha-2,4$ Kdo transferase, WaaZ (Frirdich et al., 2003). However, the core types are based mainly on the more variable outer core OS regions of E. coli LPS. The first addition to the outer core OS that is common to all core groups is the addition of glucose (Glc) to the $3^{\prime}$ position of HepII by the $\alpha 1,3$-glucosyltransferase, WaaG
(Creeger and Rothfield, 1979; Heinrichs et al., 1998b). The E. coli LPS core type R1 has been extensively studied and the enzymes involved in the construction identified (Vinogradov et al., 1999; Raetz and Whitfield, 2002). The outer core OS region of the R4 core type differs from that of $\mathrm{R} 1$ by the presence of a $\beta 1,3$ linked galactose $(\mathrm{Gal})$ residue on GlcII instead of a $\beta 1,3$ linked Glc from which the polysaccharide $\mathrm{O}$ antigen extends. These residues are transferred to the $3^{\prime}$ position of GlcII by the $\beta 1,3$ galactoyltransferase, WaaX, and the $\beta 1,3$ glucosyltransferase, WaaV, respectively (Heinrichs et al., 1998a). WaaX and WaaV are therefore important R4 and R1 core determinants. The site of polysaccharide $\mathrm{O}$ antigen attachment to the outer OS core also differs between core types. The ATP binding cassette (ABC) transporter MsbA mediates translocation of completed OS core-LPS across the IM to the site of $\mathrm{O}$-antigen ligation in the periplasm (Karow and Georgopoulos, 1993; Zhou et al., 1998; Doerrler et al., 2001).

The polysaccharide $\mathrm{O}$ antigen forms the most structurally diverse region of $E$. coli LPS with 164 groups currently identified. The $\mathrm{O}$ antigen can exist as homopolymers or heteropolymers, they can be linear or branched, and can differ in anomeric configuration and linkage positions of the different sugar residues. Generally, the $\mathrm{O}$ antigen of E. coli LPS consists of 10-25 repeating subunits containing 2-7 sugar residues (Stenutz et al., 2006). Biosynthesis of $\mathrm{O}$ antigen begins in the cytoplasm with the synthesis of the $\mathrm{O}$ antigen subunits.

The O-antigen subunits are synthesized on the lipid carrier undecaprenyl diphosphate by glycosyltransferases using sugar 
nucleotides as donors (reviewed in Samuel and Reeves, 2003). Briefly, in E. coli, there are two synthesis models for polymerization and delivery of the $\mathrm{O}$-antigen polymer to the site of ligation to OS core-LPS at the periplasmic surface of the IM. These are known as the Wzy-dependant and the ABC transporter dependant pathways, which vary in the machinery used, location of $\mathrm{O}$-antigen polymerization and manner of translocation across the IM (Whitfield, 1995). In the Wzy-dependant pathway of O antigen synthesis, the $\mathrm{O}$ antigen subunits are flipped across the IM by the putative transporter Wzx (Liu et al., 1996; Feldman et al., 1999). Polymerization of the O-antigen subunits occurs on the periplasmic surface of the IM by the polymerase Wzy which transfers the nascent polymer to the reducing end of the new subunit (Robbins et al., 1967; Marolda et al., 2006) while Wzz determines the chain length modality (extent of polymerization) (Batchelor et al., 1991; Whitfield et al., 1997). In the $\mathrm{ABC}$ transporter dependant pathway, polymerization occurs at the cytoplasmic surface of the IM by sequential addition of glycosyl residues to the non-reducing terminus of the growing polymer (Robbins et al., 1967). The $\mathrm{O}$ antigen is then transported across the IM by the $\mathrm{ABC}$ transporter composed of Wzm and Wzt (Bronner et al., 1994; Kido et al., 1995). O antigen polymers synthesized by the Wzy-dependant pathway are more common and tend to be heteropolymers with branched repeating structures while $\mathrm{O}$ antigens produced by the $\mathrm{ABC}$ transporter pathway are generally simpler linear polymers (Stenutz et al., 2006). At the periplasmic side of the IM the rough LPS and polymerized $\mathrm{O}$-antigen ligate to form LPS, a reaction catalyzed by the ligase, WaaL (Abeyrathne et al., 2005). The LPS is then ready for transport to the OM, during which certain modifications to the inner core and lipid A components may occur that impact on pathogenesis of the microorganism.

\section{Transport of LPS to the OM: LPS transport (Lpt) pathway in E. coli}

Unlike the LPS biogenesis pathway, transport of LPS through the periplasm to the OM is much less understood. Seven Lpt (LPS transport) proteins (A through G) have been implicated in transport of LPS across the periplasm to the OM. It is believed that LPS is extracted from the IM by the ABC transporter complex comprised of LptB, LptC, LptF, and LptG (Narita and Tokuda, 2009) and transferred to the periplasmic protein LptA (Sperandeo et al., 2007; Tran et al., 2008) which facilitates transfer across the periplasm to the OM assembly site composed of LptD and LptE (Wu et al., 2006; Chng et al., 2010b). The essential IM associated $\mathrm{ABC}$ transporter protein, $\mathrm{LptB}$, has been implicated in extraction of LPS from the IM (Sperandeo et al., 2006, 2007). LptB $(26.6 \mathrm{kDa})$ is predicted to contain the ATP binding fold but unlike other members of the $A B C$ transporter superfamily is soluble and does not contain a transmembrane domain, suggesting that there are other proteins that interact with LptB to form the $\mathrm{ABC}$ transporter complex. In addition, $\mathrm{LptB}$, was found in a $\sim 140 \mathrm{kDa}$ IM complex with interacting partners of unknown identity (Stenberg et al., 2005). Bioinformatic exploration identified LptF and LptG as the probable interacting partners (Ruiz et al., 2008) and LptF, LptG, and LptB were co-purified with the IM protein LptC (Narita and Tokuda, 2009) also implicated in LPS transport (Sperandeo et al., 2008). In this study, the purified
LptB/C/F/G complex was also confirmed to have ATP hydrolytic activity in vitro (Narita and Tokuda, 2009). LptC has been shown to bind LPS in vitro suggesting that LptC contains a LPS binding motif (Tran et al., 2010). Once LPS is extracted from the IM it is proposed that LptC transfers LPS to the periplasmic protein LptA which facilitates transfer of LPS to the OM assembly site LptD/E (Sperandeo et al., 2007; Tran et al., 2008). LptA is an essential soluble periplasmic protein that has been implicated in LPS transport (Sperandeo et al., 2007, 2008). LptA interacts specifically with the lipid A region of LPS and relieves LptC of LPS in vitro (Tran et al., 2008, 2010) supporting the role in LPS transport from $\mathrm{LptC}$ in the $\mathrm{LptB} / \mathrm{C} / \mathrm{F} / \mathrm{G}$ transporter in the IM to the OM assembly site LptD/E. The highly conserved and essential OMP LptD (formally known as Imp) is required for LPS transport to the cell surface (Braun and Silhavy, 2002; Bos et al., 2004) and interacts with the lipoprotein LptE forming an OM complex (Wu et al., 2006; Chng et al., 2010b). In E. coli, depletion of either LptD or LptE prevents newly synthesized LPS reaching the outer leaflet of the OM of E. coli (Wu et al., 2006) and LptE has been shown to interact specifically with LPS (Chng et al., 2010b). In E. coli, LptD is transported to the OM assembly site (the Bam complex, formerly the YaeT complex) by the periplasmic chaperone SurA where it is inserted into the OM (Wu et al., 2006; Denoncin et al., 2010).

There are two models proposed for the mechanism by which LptA facilitates the transfer of LPS from the IM ABC transporter $\mathrm{LptB} / \mathrm{C} / \mathrm{F} / \mathrm{G}$ to the $\mathrm{OM}$ assembly site LptE/F. In one model, LptA acts as a molecular chaperone that forms a soluble complex with LPS to shuttle it from the IM to the OM. This model is analogous with the Lol-mediated lipoprotein transport system where the $\mathrm{ABC}$ transporter complex LolCDE facilitates the release of lipoproteins from the IM to the periplasmic LolA that transfers the lipoproteins to the OM assembly site LolB (Tokuda, 2009). The fact that LptA binds lipid A in vitro supports the role of LptA as a periplasmic chaperone (Tran et al., 2008). In the second model, oligomers of LptA form a proteinaceous bridge spanning the periplasm by which LPS is transported from the IM to the OM assembly site. This bridge may or may not be located at membrane adhesion patches known as Bayers patches. The fact that LptA forms filaments when crystallized in the presence of LPS supports this theory (Suits et al., 2008) as does the presence of a conserved OstA domain (Pfam database http://www.sanger.ac. $\mathrm{uk} /$ cgibin/Pfam/, accession no. PF03968) in LptA that is present in the periplasmic $\mathrm{N}$-terminal of LptD (Sperandeo et al., 2006). In addition, it has been shown that LPS transport to the OM continues in spheroplasts when most of the periplasmic contents were lost (Tefsen et al., 2005). Most recently, all seven Lpt proteins have been co-purified in a membrane fraction containing both IM and OM fractions (Chng et al., 2010a). All of these data support the theory that the Lpt machinery forms a trans-envelope bridge to facilitate LPS transport from the IM to the OM.

\section{Modifications of E. coli LPS and role in pathogenesis}

Periplasmic modifications to E. coli LPS basic structure can play an important role in adaptation and pathogenesis of the microorganism (Trent et al., 2006; Raetz et al., 2007). At this stage, it is not known how these processes interact with the model for 
transport of LPS across the periplasm. Periplasmic alterations to E. coli LPS include changes to the acylation pattern and phosphate groups of lipid A. The phosphate groups attached to the disaccharide backbone of lipid A of E. coli LPS can be modified by addition of phosphate, phosphoethanolamine, (PEA) or 4-amino-4-deoxy-L-arabinose (L-Ara4N) (Zhou et al., 1999, 2000), which play a major role in susceptibility to Cationic AntiMicrobial Peptides (CAMPs) (Gunn et al., 1998; Zhou et al., 2001; Lee et al., 2004). CAMPs are important components of innate immunity as they are ubiquitous and have a broad spectrum of bacterial killing. They bind the OM of Gram-negative bacteria through electrostatic interactions with the negatively charged phosphate headgroups of lipid A, upon which they transit across the periplasm and intercalate into the IM, forming pores resulting in lysis (Zasloff, 2002). As a result, modification of the phosphate groups attached to the disaccharide back bone of LPS can significantly influence interaction with and susceptibility to CAMPs.

The addition of PEA and L-Ara4N to the phosphate headgroups of lipid A reduces the surface negative charge, increasing resistance to polymyxin B, a model CAMP (Gunn et al., 1998; Zhou et al., 2001; Lee et al., 2004). Transfer of PEA and L-Ara4N to E. coli lipid A headgroups is catalysed by the IM bound periplasmic enzymes, EptA (E. coli PEA transferase A) and ArnT (L-Ara4N transferase), respectively. EptA (formally known as PmrC in Salmonella) is a member of the alkaline phosphatase super-family that have conserved core structures and active site residues (Galperin and Jedrzejas, 2001; Trent and Raetz, 2002; Lee et al., 2004; Naessan et al., 2008). It preferentially transfers PEA to the 1- position of the disaccharide backbone of lipid A (Zhou et al., 2000; Lee et al., 2004). EptA can also catalyse the transfer of PEA to the $4^{\prime}$ position of lipid A (Zhou et al., 2000, 2001), however, this has recently been shown to only occur once decoration of the 1 phosphate group with PEA has occurred (Herrera et al., 2010) and is enhanced in the absence of ArnT (Zhou et al., 2001). Interestingly, EptA has recently been found in a protein complex with the cell division protein ZipA suggesting a role for EptA in E. coli cell division (Stenberg et al., 2005). In addition, an EptA homolog in Campylobacter jejuni has recently been found to transfer PEA to the flagellar rod promoting assembly and subsequent motility (Cullen and Trent, 2010) suggesting a larger role for this family of proteins in the decoration of bacterial surfaces. ArnT (also known as PmrK in Salmonella) preferentially transfers L-Ara $4 \mathrm{~N}$ to the $4^{\prime}$ — position of lipid A (Zhou et al., 2000, 2001; Trent et al., 2001c). Similar to EptA, ArnT has the ability to catalyse transfer of L-Ara4N to both phosphate headgroups (Zhou et al., 2000, 2001). It should also be noted that addition of L-Ara4N to the lipid A of Salmonella LPS is more prevalent than PEA addition (Zhou et al., 2001) and has greater influence over resistance to CAMPs (Tamayo et al., 2005). Transfer of an additional phosphate group to the 1-position of the disaccharide backbone of E. coli lipid A to form the 1-diphosphate structure (1-PP) is catalyzed by the undecaprenyl phosphotransferase, LpxT (Touzé et al., 2008). LpxT phosphorylation of the 1 - position of lipid A increases the negative charge of LPS and competitively inhibits transfer of PEA to the same position by EptA therefore increasing susceptibility to CAMPs (Herrera et al., 2010).
Modification of fatty acyl chain conformation of lipid A of E. coli LPS can greatly affect bacterial virulence. The OM palmitoyltransferase PagP transfers palmitate from phospholipids in the $\mathrm{OM}$ to the $2^{\prime}$ position of the proximal glucosamine residue resulting in a hepta-acylated structure (Bishop et al., 2000). Increased lipid A acylation resulting from increased PagP expression is an important mechanism for resistance to CAMPs (Guo et al., 1998) and attenuation of the inflammatory response mediated by the TLR4 signal transduction pathway resulting in immune evasion (Loppnow et al., 1986; Kawasaki et al., 2004a,b). The fatty acyl chain conformation of E. coli lipid A can be modified by the 3-O-deacylase, PagL, which removes the acyl chain from the $3^{\prime}$ position of the proximal glucosamine residue (Trent et al., 2001b). Deacylation and palmitoylation of E. coli lipid A by PagL and PagP, respectively, has been shown to decrease the potency of lipid A as an inducer of TLR4-medaited signaling (Kawasaki et al., 2004a,b). In E. coli, an acyltransferase, termed LpxP, is expressed at low temperatures $\left(12^{\circ} \mathrm{C}\right)$ and adds palmitoleate to the $2^{\prime}$ position of the distal glucosamine residue of lipid A. This enzyme effectively replaces LpxL activity and results in the addition of an unsaturated fatty acyl chain to lipid A, presumably to aid in the adjustment of the fluidity of the OM during cold shock (Carty et al., 1999). Lastly, the lipid A acyl chain conformation of $E$. coli LPS can be altered by the addition of a 2-hydroxymyristoyl group in place of myristate at the $3^{\prime}$ position of the distal glucosamine residue by the acyl transferase, LpxO (Gibbons et al., 2000).

The inner core OS of E. coli LPS can be modified by addition of PEA to the phosphate group attached to HepI and/or the $7^{\prime}$ position of KdoII (Kanipes et al., 2001; Reynolds et al., 2005; Tamayo et al., 2005). The PEA transferase EptB (E. coli PEA transferase B) catalyzes the transfer of PEA to the phosphate group attached to HepI when grown in the presence of $\mathrm{Ca}^{+2}$ (Kanipes et al., 2001; Reynolds et al., 2005). In turn, addition of PEA to KdoII of LPS appears to play a role in tolerance to elevated levels of calcium presumably by decreasing membrane permeability to the cation (Reynolds et al., 2005). The OS inner core of E. coli LPS can be modified by the addition of PEA to the $7^{\prime}$ position of KdoII (Yethon et al., 1998). In S. enterica Serovar Typhimurium, the PEA transferase, CptA, is responsible for this addition (Tamayo et al., 2005). While PEA modification of KdoII of LPS increases in polymyxin B resistant strains of S. enterica Serovar Typhimurium (Helander et al., 1994), a cptA mutant was found to be only slightly more susceptible to polymyxin B (Tamayo et al., 2005). Therefore, the function of PEA addition to KdoII of E. coli and S. enterica Serovar Typhimurium LPS is yet to be determined.

\section{REGULATION OF LPS BIOGENESIS AND PERIPLASMIC PROTEIN FOLDING IN $E$. coli}

There are a number of regulatory systems that act in concert to tightly regulate periplasmic protein folding and the biosynthesis of LPS in E. coli (Figure 4). The two component system is the most prevalent form of signal transduction enabling bacteria to alter cellular behavior in response to environmental cues (Stock et al., 2000). Typically, a two component system consists of a sensor histidine kinase that responds to specific signals by altering 


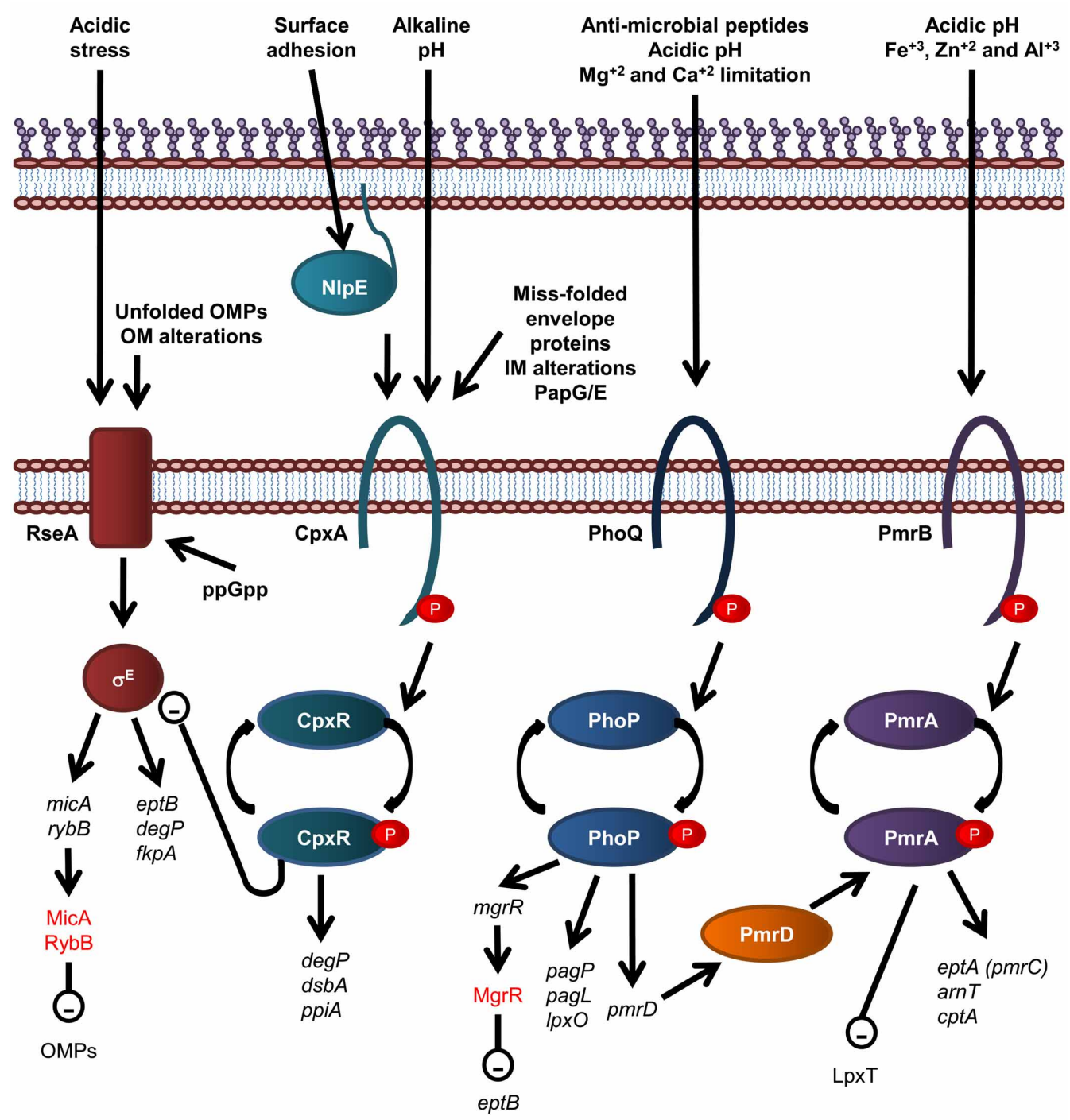

FIGURE 4 | Overview of regulatory systems involved in LPS biogenesis and periplasmic protein folding in $\boldsymbol{E}$. coli. The sensor histidine kinase and cognate response regulator protein of each of the $\mathrm{CpxR} / \mathrm{A}, \mathrm{PhoP} / \mathrm{Q}$, and PmrA/B two component systems are shown. The $\sigma E$ envelope stress response is also shown, where $\sigma \mathrm{E}$ activation is inversely related to stability of the IM anti-sgma factor, RseA. Genes are in black text, lower case and italicized while proteins and sRNAs are in sentance case. sRNAs are in red text. Up-regulation of down stream processes is indicated by arrows while down-regulation is indicated by a minus sign within a circle. the phosphorylated state of a cognate response regulator protein. The vast majority of response regulators are DNA-binding transcription factors whose affinities for their target promoters are modulated by phosphorylation. Thus, by altering the phosphorylated state of a response regulator, a signal often modifies the gene expression profile of an organism (Beier and Gross, 2006; Mascher et al., 2006).

Modification of E. coli LPS is primarily under the control of the PmrA/B and PhoP/Q two component signal transduction systems, which respond to environmental cues that reflect that of the macrophage phagosome, such as low $\mathrm{pH}$ and the presence of antimicrobial peptides. The resulting modifications lead to enhanced survival in both host and non-host environments (Gunn et al., 1998). For example, in S. enterica serovar
Typhimurium and E. coli, the substitution of lipid A headgroups with PEA and L-Ara4N, by EptA and ArnT, respectively, and the addition of PEA to KDOII of LPS by CptA, is under the control of the PmrA/PmrB two component histidine kinase/response regulator pair (Gunn et al., 1998; Zhou et al., 2001; Lee et al., 2004; Tamayo et al., 2005) which responds to mild acidic conditions and the presence of $\mathrm{Fe}^{+3}, \mathrm{Zn}^{+2}$, and $\mathrm{Al}^{+3}$ (Zhou et al., 1999, 2000; Trent et al., 2001a; Gibbons et al., 2005). These modifications lead to enhanced resistance to CAMPs (Helander et al., 1994; Gunn et al., 1998; Zhou et al., 2001; Lee et al., 2004). In addition, growth conditions that promote PmrA activation inhibit LpxTdependent phosphorylation of lipid A, therefore promoting resistance to CAMPs, suggesting that PmrA/B regulates LpxT expression, although this effect does not involve the control of LpxT 
transcription (Herrera et al., 2010). The expression of PagP, PagL, and $\mathrm{LpxO}$ is under the control of the PhoP/Q two component histidine kinase/response regulator system in S. Typhimurium (Belden and Miller, 1994; Trent et al., 2001b; Kawasaki et al., 2004a) which is activated by the presence of CAMPs, low $\mathrm{pH}$ and $\mathrm{Mg}^{+2}$ and $\mathrm{Ca}^{+2}$ limitation (Garcia Vescovi et al., 1996; Soncini et al., 1996; Bader et al., 2003, 2005). To add another layer of complexity, the PhoP activated PmrD protein controls the PmrA/B two component system at a post-translational level as PmrD binds to phosphorylated PmrA, preventing dephosphorylation, leading to PmrA-mediated transcription (Kox et al., 2000; Kato and Groisman, 2004). Through this mechanism, the presence of CAMPs can be detected by the PhoQ sensory histidine kinase and lead to the PmrA-mediated transcription of genes; eptA, arnT, and $c p t A$, as well as the PhoP-mediated transcription of pagP, which leads to LPS modification by EptA, ArnT, CptA, and PagP and subsequent increased resistance to CAMPs (Guo et al., 1997).

An alternative two component system that has been implicated in regulation of LPS modification is the SoxR/S superoxide and nitric oxide sensing two component system (Lee et al., 2009). The cognate response regulator, SoxS, binds the waaY promoter, leading to expression of the WaaY protein, which is responsible for phosphorylating the HepII of E. coli LPS inner core. WaaY expression is also regulated by the Multiple-Antibiotic Resistance marker, MarA, and is induced by salicylate, suggesting a role in antimicrobial resistance. In support of this, phosphorylation of the inner OS core of E. coli LPS has been implicated in conferring enhanced resistance against multiple drugs, possibly by increasing the integrity of the OM through cross-linking of neighboring LPS molecules via binding of divalent cations (Yethon et al., 1998; Yethon and Whitfield, 2001; Lee et al., 2009).

Recently, the PhoP/Q two component system has been shown to activate expression of the $\mathrm{Mg}^{+2}$-responsive RNA, MgrR, which down regulates expression of EptB (Moon and Gottesman, 2009), which provides an explanation as to why EptB-mediated PEA addition to $E$. coli LPS is increased in the presence of $\mathrm{Ca}^{+2}$, which has been shown to inhibit activation of the PhoP/Q two component system (Garcia Vescovi et al., 1996). Small non-coding RNAs (sRNAs), such as MgrR, are fast becoming recognized as key components of regulatory circuits that act as a fast acting "switches" to respond to stress signals (reviewed in Waters and Storz, 2009). The sRNAs, MicA and RybB, are activated in response to the $\sigma^{\mathrm{E}}$-envelope stress signaling to down regulate OMPs (Papenfort et al., 2006; Moon and Gottesman, 2009; Overgaard et al., 2009). Interestingly, the $\sigma^{\mathrm{E}}$-envelope stress response has been implicated in upregulation of EptB expression (Figueroa-Bossi et al., 2006), suggesting the possibility that MgrR participates in a negative feedback loop that controls EptB-mediated modification of LPS (Overgaard et al., 2009). The $\sigma \mathrm{E}$ envelope stress signal system detects OM protein misfolding (Mecsas et al., 1993; Missiakas et al., 1996) and is activated by the well known cytoplasmic nutritional stress signal, guanosine $3^{\prime}, 5^{\prime}$-bis-pyrophosphate (ppGpp) (Costanzo and Ades, 2006).

Periplasmic protein folding and degradation is controlled by the CpxR/A two component system (reviewed in Vogt and
Raivio, 2012), which senses miss-folded envelope proteins, Pap pilus sub-unit over-expression, alkaline $\mathrm{pH}$, and alterations of the IM (Nakayama and Watanabe, 1995; Jones et al., 1997; Mileykovskaya and Dowhan, 1997; Danese and Silhavy, 1998). CpxA is also activated by surface adhesion detected by the OM lipoprotein NlpE (Otto and Silhavy, 2002). The cognate response regulator, $\mathrm{CpxR}$, induces the expression of periplasmic protein folding factors such as the oxidoreductase DsbA and the peptidyl-prolyl isomerize PpiA, and the periplasmic protease and chaperone DegP (Danese et al., 1995; Danese and Silhavy, 1997; Pogliano et al., 1997). Interestingly, CpxR negatively and directly regulates the expression of the rpoErseABC operon, which encodes the alternative sigma factor $\sigma^{\mathrm{E}}$, the mediator of an additional envelope stress response that detects OM protein misfolding (De Wulf et al., 2002; Price and Raivio, 2009).

\section{THE GRAM-NEGATIVE CELL ENVELOPE OF $\boldsymbol{N}$. meningitidis}

N. meningitidis has a Gram-negative envelop which is structurally analogous to that of E. coli. However, there are some fundamental differences in the biology of these two pathogens. For example, unlike E. coli and other Gram-negative bacteria, LPS deficient strains of N. meningitidis are viable (Steeghs et al., 1998) which has led to significant advancements in the understanding of the machinery involved in LPS biosynthesis, transport, and assembly in the OM. Conversely, it has been recently shown that the protein isomerization pathway in N. meningitidis is essential for cell viability (Kumar et al., 2011), whilst this is not the case for E. coli. These observations suggest there are some important differences to be found in the genesis of the OM in Neisseria sp. Therefore, the remainder of this review will detail the differences in the oxidation/isomerization pathways and LOS biosynthesis pathway in Neisseria $s p$.

\section{THE ROLE OF THE PERIPLASMIC OXIDOREDUCTASES IN OM BIOGENESIS AND PATHOGENESIS OF $\boldsymbol{N}$. meningitidis}

In $E$. coli, the oxidation pathway is a generalist one recognizing all chromosomally encoded proteins that require disulfide bonds and is supplemented with specialist protein oxidation pathways usually encoded on genetic islands or plasmids, while the isomerization pathway contains a generalist isomerase $E c D s b C$ and two substrate specific isomerases, $E c$ DsbG and $E c D s b E$. In contrast, $N$. meningitidis appears to have adopted a specialist protein oxidation pathway which is partnered with a diversified protein isomerization pathway containing a wide variety of partners that interact with $N m D s b D$. Specifically, N. meningitidis contains three DsbA homologs (NmDsbA1, NmDsbA2, and $N m$ DsbA3) as well as a single copy of $N m$ DsbB, $N m$ DsbC and NmDsbD (Figure 5) (Sinha et al., 2004, 2008; Tinsley et al., 2004). These pathways have been shown to make important contributions to the virulence, pathogenesis, and viability of this microorganism.

\section{Oxidation pathway of periplasmic protein folding in $\mathbf{N}$. meningitidis}

Each of the meningococcal DsbA enzymes have been shown to play a central role in virulence and the presence of at least one DsbA is required for normal growth of $N$. meningitidis (Tinsley 


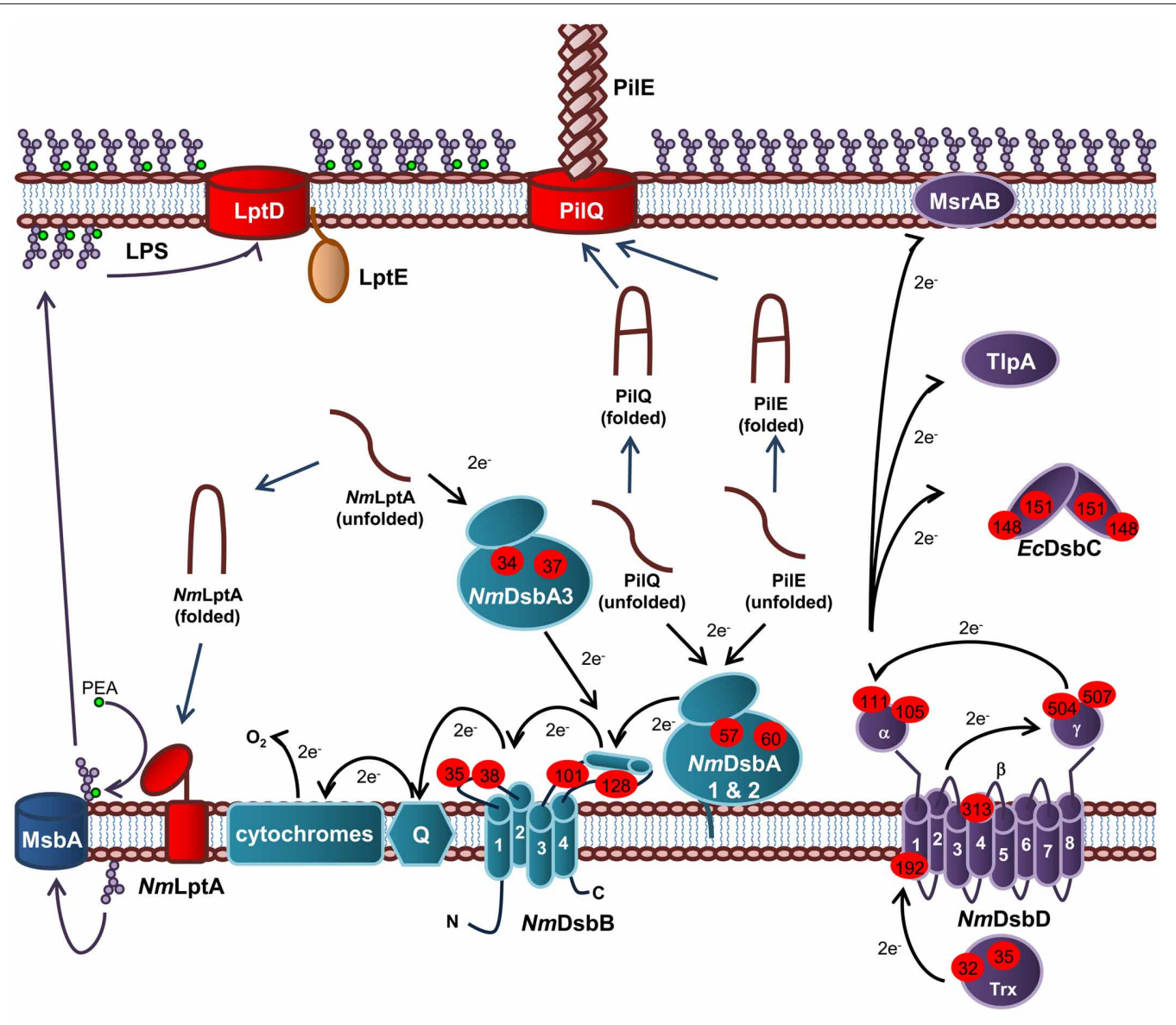

\section{Oxidation}

FIGURE 5 | Oxidation and isomerization pathways of periplasmic protein folding in $\boldsymbol{N}$. meningitidis and contribution to virulence. Oxidation pathway: The oxidoreductases NmDsbA1 and NmDsbA2 and IM bound lipoproteins that catalyze disulfide bond formation in the PilE and PilQ subunits of the Type IV secretion system. The soluble preiplasmic oxidoreductase $N m D s b A 3$ catalyzes disulfide bond formation in the periplasmic domain of the PEA transferase NmLptA, which is responsible for PEA modification of LOS lipid A upon translocation across the IM by the transporter, MsbA, prior to transport across the periplasm and integration into the OM by LptD and LptE. NmDsbA1, NmDsbA2, and NmDsbA3 are re-oxidized by the IM bound
$N m D s b B$. Under aerobic growth conditions, electrons flow from EcDsbB to molecular oxygen via ubiquinone $(\mathrm{Q})$ and cytochrome. Isomerization pathway: The isomerization pathway consists of a soluble periplasmic $\mathrm{NmDsbC}$, for which there is no known substrate and an IM bound NmDsbD, which has also been implicated in the reduction of TIpA and MsrAB. NmDsbD is reduced by thioredoxin in the cytoplasm. The oxidation pathway is in turquoise and the isomerization pathway is in purple. Cysteine residues are denoted by a red circle containing the residue number. The direction of electron transfer is shown by black arrows while protein folding reactions are shown by dark blue arrows and cellular processes are shown by dark purple arrows. et al., 2004; Sinha et al., 2008; Piek et al., 2012). The presence of $N m$ DsbA1 or $N m$ DsbA2 is required for formation of functional Type IV pilin and normal growth of $N$. meningitidis in the presence of reducing agents (Tinsley et al., 2004; Sinha et al., 2008). Type IV pili is a key virulence determinant of $N$. meningitidis as it initiates attachment to host cells and mediates DNA binding and uptake during natural transformation. Both NmDsbA1 and NmDsbA2 have been shown to introduce disulfide bonds into PilQ, the secretin which forms the pore through which the Type IV pili are extruded (Sinha et al., 2008). In addition, both oxidoreductases have been implicated in oxidation of the Type IV pili filament subunit, PilE (Tinsley et al., 2004). Recently, NmDsbA3 has been shown to catalyze disulfide bond formation in the LPS PEA transferase, NmLptA (Piek et al., 2012) and has therefore been implicated in resistance to CAMPs in N. meningitidis. Interestingly, $N m \mathrm{DsbA3}$ is unable to compensate for the loss of NmDsbA1 and NmDsbA2 (Tinsley et al., 2004; Sinha et al., 2008) and conversely, NmDsbA1 and NmDsbA2 are unable to compensate for the lack of NmDsbA3 (Piek et al., 2012), suggesting that these enzymes are able to recognize and discriminate between substrates. The meningococcal DsbA enzymes were also found to preferentially interact with different $E c D s b A$ substrates when used to complement the E. coli $\Delta d s b A$ mutant strain JCB571 (Sinha et al., 2004). While all three enzymes were able to restore resistance to the reducing agent dithiothreitol (DTT) only NmDsbA1 restored motility to JCB571 and only $N m$ DsbA2 restored alkaline phosphatase activity (Sinha et al., 2004). However, NmDsbA3 was able to oxidize FlgI and restore 
motility when over-expressed (Vivian et al., 2008). All three of the oxidoreductases have been shown to have oxidoreductase activity in vitro (Vivian et al., 2008, 2009; Lafaye et al., 2009).

Unlike EcDsbA, NmDsbA1 and NmDsbA2 are membrane bound lipoproteins which share $78 \%$ amino acid identity with each other. In contrast, $N m D s b A 3$ is a soluble periplasmic oxidoreductase sharing $57 \%$ and $51 \%$ amino acid identity with $N m$ DsbA1 and NmDsbA2, respectively (Sinha et al., 2004; Tinsley et al., 2004). All three of the meningococcal oxidoreductases share around $\sim 20 \%$ amino acid identity with $E c \mathrm{DsbA}$ (Sinha et al., 2004). Crystals of the soluble domain of NmDsbA1 [PDB3A3T (Vivian et al., 2009) and PDB 3DVW (Lafaye et al., 2009)] and NmDsbA3 [PDB 2ZNM (Vivian et al., 2008) and PDB 3DVX (Lafaye et al., 2009)] have been solved revealing a similar tertiary structure to $E c \mathrm{DsbA}$ with a thioredoxin domain containing the thioredoxin-like fold and the active site motif CXXC with an inserted $\alpha$-domain. Uniquely, $N m$ DsbA3 contains an active site motif of CVHC in an open loop conformation (Vivian et al., 2008) while NmDsbA1 and NmDsbA2 (modeled on $\mathrm{N} m \mathrm{DsbA}$ ) contain the canonical active site of $\mathrm{CPHC}$ located at the $\mathrm{N}$-terminus of the first $\alpha$-helix of the thioredoxin domain, which is a similar in arrangement to $E c$ DsbA (Lafaye et al., 2009; Vivian et al., 2009). All three enzymes are extremely oxidizing with a standard redox potential of $\sim-80 \mathrm{mV}$ (Vivian et al., 2008, 2009; Lafaye et al., 2009) compared to that of EcDsbA ( -120 mV) (Huber-Wunderlich and Glockshuber, 1998). The Thr residue immediately preceding cis-Pro residue corresponding to position 151 in $E c \mathrm{Dsb}$, has been shown to confer this higher oxidizing power of these enzymes (Lafaye et al., 2009; Ren et al., 2009).

\section{Isomerization pathway of periplasmic protein folding in N. meningitidis}

Unlike E. coli, the isomerization pathway of N. meningitidis is essential (Kumar et al., 2011) suggesting a fundamental difference in this biochemical pathway and a key role in meningococcal lifestyle and virulence. $N$. gonorrhoeae contains a periplasmic thioredoxin-like protein, TlpA, which influences susceptibility to oxidative stress and the ability to survive in cervical epithelial cells (Achard et al., 2009). It has been suggested that TlpA is kept in a reduced state by DsbD (Achard et al., 2009). The substrate of TlpA is not known but is thought to be the methionine sulfoxide reductase (MsrAB), which repairs methioinine residues which have been attacked by reactive oxygen species (ROS). MsrAB is unusually located in the OM of $N$. gonorrhoeae, instead of in the cytoplasm as it is in other Gram-negative bacteria (Skaar et al., 2002). However, other work in this area has proposed that MsrAB is itself a direct redox partner of $\mathrm{NmDsbD}$ (Brot et al., 2006). Therefore, in Neisseria sp. there has been a diversification of redox partners for $N m D s b D$, all of which relate to the repair of proteins damaged due to oxidative stress.

\section{BIOSYNTHESIS AND TRANSPORT OF LOS TO THE OM OF N. meningitidis}

In contrast to E. coli LPS, N. meningitidis does not produce an endotoxin with repeating $\mathrm{O}$-antigen and is therefore more appropriately named lipooligosaccharide (LOS) (Jennings et al., 1980; Tsai et al., 1987). Meningococcal LOS consists of an inner OS core region attached to lipid $\mathrm{A}$ and a variable outer OS core region ( $\alpha$-chain) (Figure 6). The inner OS core consists of two Hep residues and two Kdo residues that can be decorated with variable sugar additions. Biosynthesis of meningococcal LOS is initiated in the cytoplasm with synthesis of the inner OS core attached to lipid A. The variable $\alpha$-chain is then synthesized by sequential addition of sugars to HepI of the inner core and the completed LOS is then translocated to the periplasmic surface of the IM, transported across the periplasm and integrated into the OM.

\section{Biosynthesis of meningococcal LOS}

Biosynthesis of the $\mathrm{Kdo}_{2}$-lipid A region of $\mathrm{N}$. meningitidis LOS is similar to the biogenesis of E. coli LPS except that one lauroyl residue is transferred to the $2^{\prime}$ position of each glucosamine unit resulting in a symmetrical molecule (Kulshin et al., 1991, 1992). Meningococcal LOS is synthesized by sequential transfer of sugars to the $\mathrm{Kdo}_{2}$-lipid A structure at the cytoplasmic surface of the IM. There are many enzymes known to facilitate this process.

The basic OS core region of meningococcal LOS consists of $\mathrm{Glc}_{1} 1,4\left(\mathrm{GlcNAc}_{1}\right)-\mathrm{Hep}_{2}-\mathrm{Kdo}_{2}$ attached to lipid A (Kahler and Stephens, 1998). Transfer of a ADP-Hep to KdoI of the conserved $\mathrm{Kdo}_{2}$-lipid A structure, mediated by the $\alpha 1,5$ heptosyltransferase, $\mathrm{RfaC}$, forms $\mathrm{Hep}_{1}-\mathrm{Kdo}_{2}$-lipid A. The $\alpha 1,3$ heptosyltransferase, $\mathrm{RfaF}$, then catalyzes the transfer of the second ADP-Hep to HepI to form $\mathrm{Hep}_{2}-\mathrm{Kdo}_{2}$-lipid A (Kahler and Stephens, 1998) which forms the minimum LOS structure required for invasion of nasopharyngeal epithelial cell lines (Plant et al., 2006). The structure of meningococcal LOS then diverges from the E. coli model by addition of $\mathrm{N}$-Acetylglucosamine (GlcNAc) to the $2^{\prime}$ position of HepII and Glc to the $4^{\prime}$ position of HepI (Kahler et al., 1996b). The transfer of UDP-GlcNAc to position $2^{\prime}$ of HepII is catalyzed by the $\alpha 1,2 \mathrm{~N}$-acetylglucosamine transferase, RfaK. This step results in the formation of $\mathrm{GlNAc}_{1}-\mathrm{Hep}_{2}-\mathrm{Kdo}_{2}$-lipid $\mathrm{A}$ and is a necessary prerequisite for further LOS biosynthesis (Kahler et al., 1996a). The transfer of UDP-Glc to the $4^{\prime}$ position of HepI is mediated by the $\beta 1,4$ glycosyltransferase, LgtF, resulting in the conserved core region of meningococcal LOS, Glc $\beta 1,4$ $\left(\mathrm{GlNAc}_{1}\right)-\mathrm{Hep}_{2}-\mathrm{Kdo}_{2}$-lipid A (Kahler et al., 1996b). From the terminal Glc of this structure the variable $\alpha$-chain can extend.

Meningococcial LOS can express a long $\alpha$-chain composed of lacto- $N$-neotetraose $\quad($ Gal $\quad \beta 1,4 \rightarrow$ GlcNAc $\beta 1,3 \rightarrow$ Gal $\beta 1,4 \rightarrow$ or LNT) or a short $\alpha$-chain composed of either Gal $\alpha 1,4 \rightarrow \mathrm{Gal} \beta 1,4 \rightarrow$ or Gal $\beta 1,4 \rightarrow$ and is dependent on expression of genes in the lipooligosaccharide glycosyltransferase (lgt $A B C E)$ locus (Wakarchuk et al., 1996). Specifically, switching between the $\alpha$-chains is achieved by high frequency mutation within the poly$\mathrm{G}$ tract of $\lg t A$ and $\lg t C$, a process referred to as phase variation (Jennings et al., 1999; Zhu et al., 2002). First, the $\beta 1,4$ galactosyltransferase, LgtE, catalyzes transfer of UDP-Galactose to the terminal Glc attached to HepI. LNT formation then depends on the phase variable expression of the $\beta 1,3 \mathrm{~N}$-acetylglucosamine transferase, LgtA, that transfers UDP-GlcNAc to the $3^{\prime}$ position of the terminal Gal of Gal $\beta 1,4 \rightarrow \mathrm{Glc} \beta 1,4 \rightarrow \mathrm{HepI}$ of LOS. The $\beta 1,4$ galactosyltransferase, LgtB, then transfers UDP-Galactose to the $4^{\prime}$ position of the GlcNAc resulting in the LNT structure (Jennings et al., 1995; Wakarchuk et al., 1996). In the absence of $\operatorname{lgt} A$ expression, the $\alpha$-chain can terminate at Gal $\beta 1,4 \rightarrow$ or 


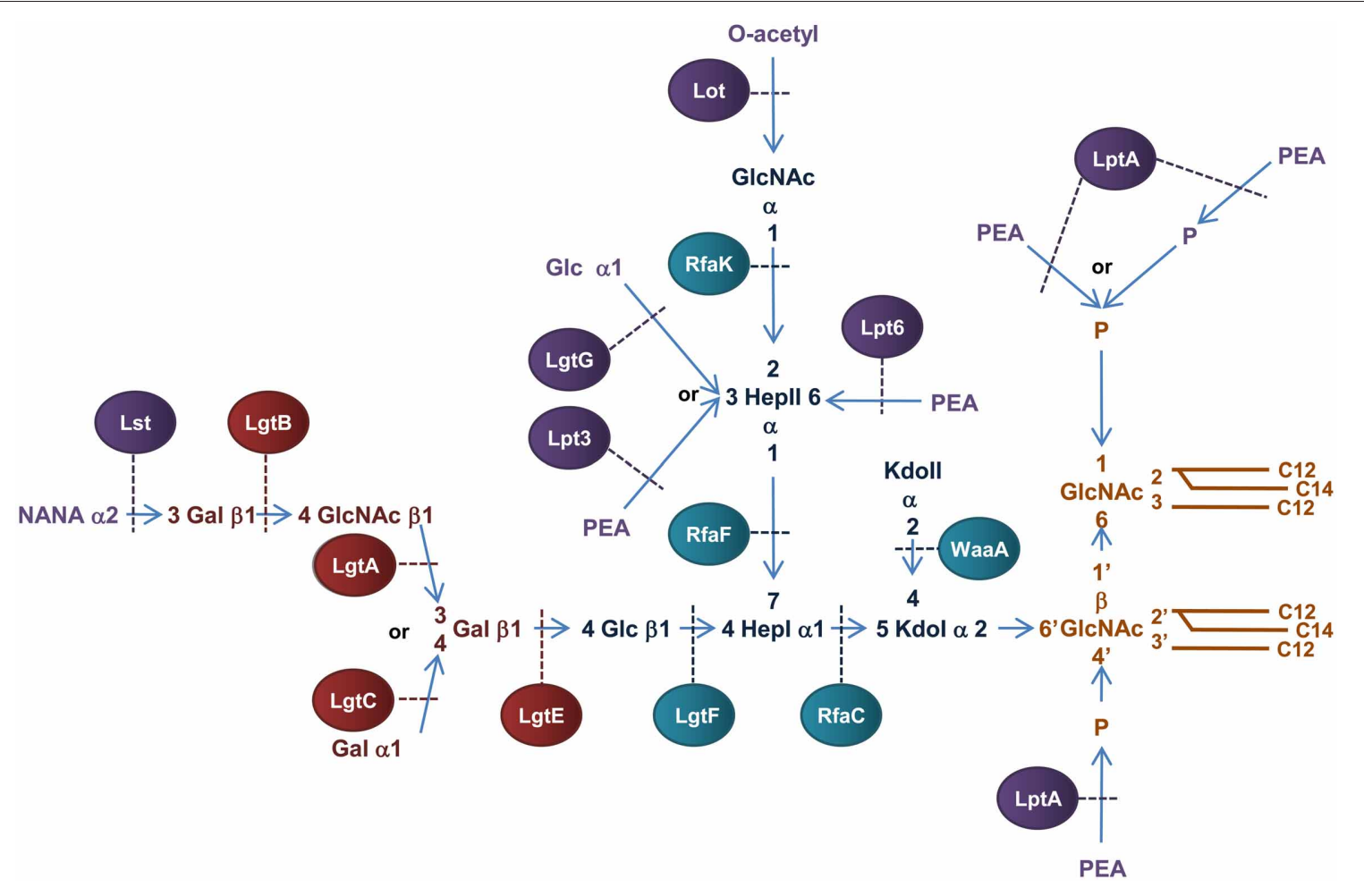

FIGURE 6 | Structure and biosynthesis of $\boldsymbol{N}$. meningitidis LOS. Conserved Lipid A region is in orange while the conserved inner OS core region is in dark blue and the variable $\alpha$-chain in maroon. Variable modifications to these regions are in purple. Glycosyltransferases that form the inner OS core backbone and $\alpha$-chain are denoted by the blue and light maroon boxes, respectively. Enzymes that modify the structure are in purple. The number of carbons present in each acyl chain is denoted by $\mathrm{C}$ followed by a number. Structure and biosynthesis of the lipid A region is reviewed in detail elsewhere (Raetz and Whitfield, 2002). Refer to text for details on enzyme functions. depending on the phase variable expression of $\lg t C$, the $\alpha 1,4$ galactosyltransferase LgtC can transfer UDP-Galactose to the $4^{\prime}$ position of the terminal Gal residue resulting in the formation of the short $\alpha$-chain, Gal $\alpha 1,4 \rightarrow \operatorname{Gal} \beta 1,4 \rightarrow$ (Wakarchuk et al., 1998).

Variable additions to the LOS inner OS core can occur prior to transport across the IM. The terminal GlcNAc attached to the $2^{\prime}$ position of HepII can be O-acetylated by the phase variable lipooligosaccharide $\mathrm{O}$-acetyltransferase, Lot, forming the $\gamma$-chain extension (Kahler et al., 2006) and the O-3 position of HepII can be modified by transfer of a Glc residue mediated by the $\alpha 1,3$ glycosyltransferase, LgtG (Banerjee et al., 1998). The lgtG gene is phase variable (Jennings et al., 1999) but is part of a small genetic island that is absent in some isolates (Jennings et al., 1999).

\section{Transport of LOS to the OM: Lpt pathway in N. meningitidis}

Meningococcal LOS is transported across the IM by the IM ABC transporter MsbA (Karow and Georgopoulos, 1993; Zhou et al., 1998; Doerrler et al., 2001) to the periplasm and is targeted to the OM by Lpt proteins similar to that in E. coli. One notable difference is that in N. meningitidis, LptE is not essential to LOS transport and has been suggested to have a chaperone-like role in LptD biogenesis (Bos and Tommassen, 2011). Interestingly, unlike E. coli where the periplasmic chaperone SurA is required for transport of LptD to the OM (Vertommen et al., 2009; Denoncin et al., 2010), SurA appears not to play a role in
OM biogenesis of $N$. meningitidis (Volokhina et al., 2011). Upon integration into the OM, meningococcal LOS can be modified by the transfer of $N$-acetylneuraminic acid (NANA) to the $3^{\prime}$ position of the terminal Gal of LNT by the LOS $\alpha 2,3$ sialyltransferase, Lst, resulting in an $\alpha$-chain extension of $\mathrm{NANA} \alpha 1,3 \rightarrow \mathrm{Gal} \beta 1,4 \rightarrow \mathrm{GlCNAc} \rightarrow \beta 1,3 \rightarrow \mathrm{Gal} \beta 1,4 \rightarrow \quad$ (Mandrell et al., 1991; Gilbert et al., 1996).

\section{Periplasmic modifications of meningococcal LOS}

The lipid A and inner core regions of meningococcal LOS can be modified by the addition of PEA during transit through the periplasm to the OM. The OS core of meningococcal LOS can be modified by addition of PEA to the $3^{\prime}$ or $6^{\prime}$ positions of HepII catalysed by the enzymes, Lpt3 (LOS PEA transferase 3 ) and Lpt6 (LOS PEA transferase 6), respectively (Mackinnon et al., 2002; Wright et al., 2004; Kahler et al., 2005). Transfer of PEA to the $3^{\prime}$ carbon of HepII by Lpt3 can be competitively inhibited by the addition of $\alpha 1-3$ Glc to the same position by LgtG in the cytoplasm before transport into the periplasm (Banerjee et al., 1998; Mackinnon et al., 2002). In addition, acetylation of the terminal GlcNAc attached to the $2^{\prime}$ position of HepII in conjunction with an $\alpha$-chain of LNT can inhibit PEA addition to the $3^{\prime}$ position of HepII (Kahler et al., 2006). Unlike E. coli and Salmonella, the lipid A phosphate headgroups of meningococcal LOS are not decorated with arabinose but exist as a mixture of "phosphoforms." 
The basal diphosphorylated species of meningococcal LOS can be modified by the addition of a single PEA to the $4^{\prime}$ headgroup or by addition of PEA to both ends of the disaccharide backbone (never at the 1 position alone). The lipid A can also contain an additional phosphate residue at the 1 headgroup with or without PEA (Kulshin et al., 1992; Cox et al., 2003). In N. meningitidis, the PEA transferase NmLptA catalyzes the transfer of PEA to the lipid A of LOS (Cox et al., 2003). While inactivation of NmlptA results in a total loss of PEA substitutions to lipid A, it is not known whether NmLptA transfers one or both of the PEA groups to the lipid A headgroups (Cox et al., 2003).

\section{Role of LOS in pathogenesis of $N$. meningitidis}

The length and composition of the LOS $\alpha$-chain is a major virulence determinant of $N$. meningitidis. Invasive isolates of $N$. meningitidis express an $\alpha$-chain of LNT that mimics human paragloboside, increasing resistance to serum bactericidal activity (Moran et al., 1996; Harvey et al., 2001). An $\alpha$-chain of LNT is therefore required for survival in the blood stream and the establishment of bacteraemia (Mackinnon et al., 1993; Moran et al., 1994; Virji et al., 1995). Sialylation of the $\alpha$-chain further increases resistance to serum bactericidal activity (Kahler et al., 1998). In contrast, a short $\alpha$-chain consisting of Gal $\alpha 1,4 \rightarrow \mathrm{Gal} \beta 1,4 \rightarrow$ or $\beta 1,4 \mathrm{Gal} \rightarrow$ allows colonization and invasion of the nasopharynx by invasive isolates (Moran et al., 1994). The ability to switch between carriage and invasive modes by the phase variable expression of $\alpha$-chain biosynthesis genes $\operatorname{lgt} A$ and $\operatorname{lgt} C$ is therefore a major virulence determinant of $N$. meningitidis.

Periplasmic modifications of the lipid A and OS core of meningococcal LOS by PEA can mediate resistance to defensins at the site of colonization and attachment to host cells as well as influence survival in the blood stream and the potency of lipid $\mathrm{A}$ as an inducer of the host inflammatory immune response (Kahler et al., 1998; Ram et al., 2003; Tzeng et al., 2005; Takahashi et al., 2008). NmLptA catalyzed addition of PEA to lipid A of meningococcal LOS is essential for resistance to CAMPs (Tzeng et al., 2005) and the ability to attach to nasopharyngeal epithelial cell lines (Takahashi et al., 2008). NmLptA mediated addition of PEA groups to lipid A of LOS of the closely related pathogen $N$. gonorrhoeae results in increased resistance to complement mediated killing by normal human serum (Lewis et al., 2009), suggesting a possible role of PEA addition to lipid A in meningococcal serum resistance. In addition, the level of PEA decoration of lipid A can influence the potency of lipid $\mathrm{A}$ as an inducer of the host inflammatory immune response (Liu et al., 2010). Variable sugar additions to the carbons of HepII of the inner OS core of meningococcal LOS can influence resistance to complement mediated lysis and therefore play an integral role in meningococcal pathogenesis. The PEA groups attached to HepII of meningococcal LOS are a target for complement component $\mathrm{C} 4 \mathrm{~b}$ resulting in enhanced killing by the classical pathway of complement. Furthermore, the O-6 linked PEA groups are more efficient at binding the complement component, C4b, than the O-3 linked PEA (Ram et al., 2003). As a result modification of the inner OS core of meningococcal LOS, particularly decoration with PEA, is central to pathogenesis of N. meningitidis.

\section{REGULATION OF LOS BIOGENESIS AND PERIPLASMIC PROTEIN FOLDING IN $\boldsymbol{N}$. meningitidis}

In contrast to E. coli, which encodes more than 30 two component systems (Oshima et al., 2002; Yamamoto et al., 2005), N. meningitidis contains only four predicted two component systems which is a feature common to obligate host pathogens from restricted environments (Parkhill et al., 2000; Tettelin et al., 2000). The two component system, MisR/MisS (meningococcal inner core structure), has been shown to be involved in LOS modification and periplasmic protein folding (Figure 7). The MisR/MisS histidine kinase/response regulator pair has been proposed to be analogous to the PhoP/PhoQ system of E. coli (Johnson et al., 2001; Newcombe et al., 2005). However, evidence for responses to different environmental cues suggests a different role. Inactivation of the MisR/MisS two component system

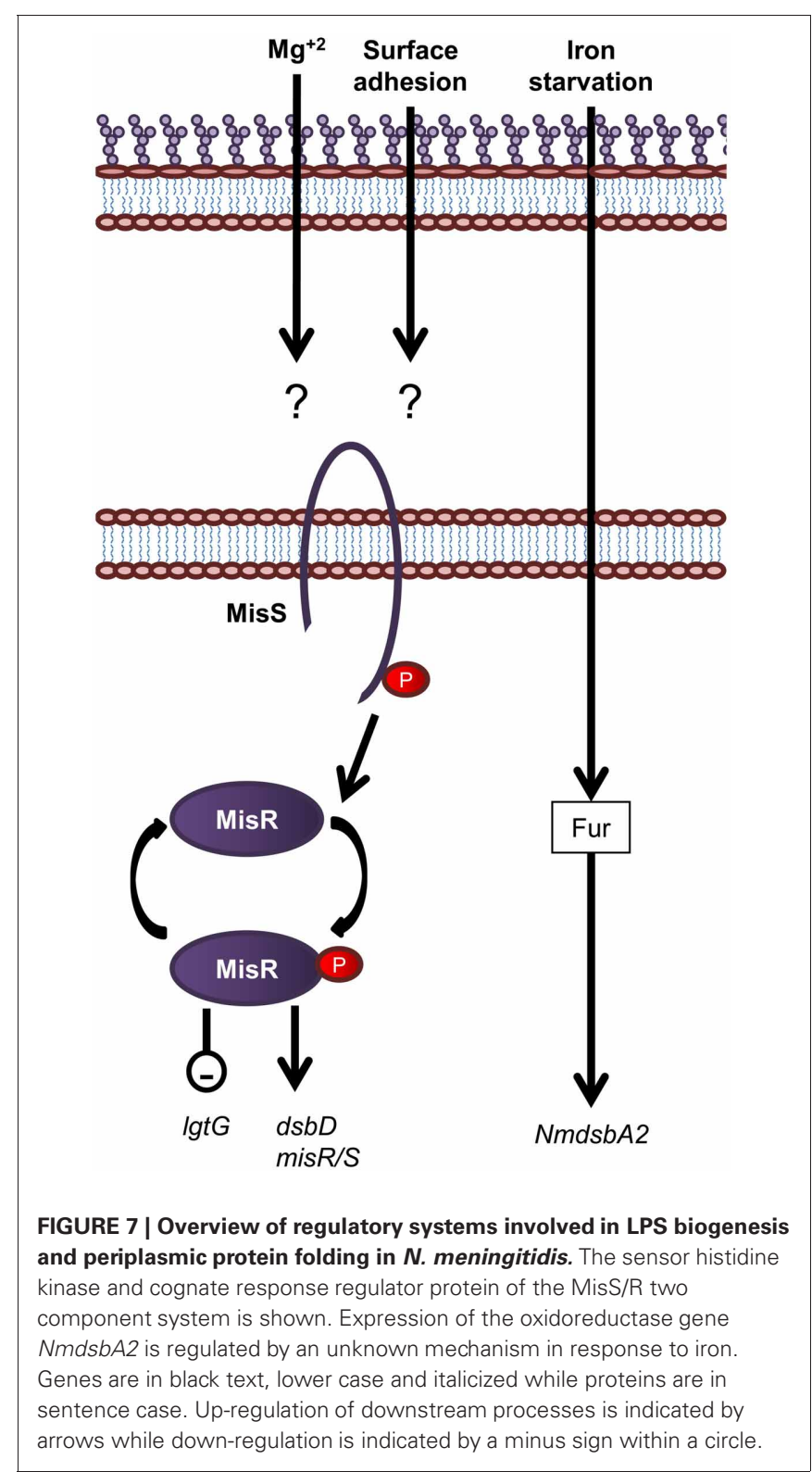


in N. meningitidis increases sensitivity to CAMPs (Johnson et al., 2001; Tzeng et al., 2004), reduces haem utilization (Zhao et al., 2010) and causes attenuation in a mouse model of meningococcal infection (Newcombe et al., 2004; Rustam et al., 2006). While initial studies using N. meningitidis serogroup C strains, M96255789 and L91543, found the MisR/MisS two component system to be activated by $\mathrm{Mg}^{+2}$ limitation (Johnson et al., 2001; Newcombe et al., 2005), this has not been confirmed by more recent studies in N. meningitidis serogroup B strain, NMB (Tzeng et al., 2006). In addition, a separate study has shown that MisR/MisS is regulated in response to host cell contact (Jamet et al., 2009). The MisR/MisS two component system influences decoration of the inner OS core of meningococcal LOS. Inactivation of MisR resulted in increased expression of the LOS glycosyltransferase, LgtG, responsible for transferring a Glc residue to the 0-3 position of HepII (Tzeng et al., 2004). In addition, while there was no change in expression of the inner core PEA transferase enzymes, Lpt3 and Lpt6, the MisR mutant was completely devoid of PEA modifications of the inner core, which in turn resulted in increased sensitivity to CAMPs (Tzeng et al., 2004). In addition, transcription of $d s b D$, but not $d s b C$ or other $d s b$ genes, is positively regulated by the MisR/MisS (Tzeng et al., 2008; Kumar et al., 2011). Apart from the MisR/MisS circuit, the only other known regulatory circuit for the protein oxidation pathway is the fur (ferric uptake regulator) dependent regulation of $\mathrm{NmdsbA2}$ (Klee et al., 2000), which is upregulated in conditions of iron starvation (Grifantini et al., 2003).

Extensive transcriptome analysis of gene expression in $N$. meningitidis has not revealed any other regulatory circuits controlling the protein oxidation and isomerization or LOS biosynthesis pathways in response to specific conditions such as heat shock (Guckenberger et al., 2002), contact with eukaryotic cells (Grifantini et al., 2002a,b; Dietrich et al., 2003), exposure to human serum (Dietrich et al., 2003; Kurz et al., 2003), biofilm formation (Phillips et al., 2012), oxidative stress (Seib et al., 2007), or iron starvation (Grifantini et al., 2003; Basler et al., 2006). In addition, genes involved in LPS biogenesis and periplasmic protein folding are not regulated in response to the alternative sigma factors, $\sigma^{\mathrm{E}}$ (Gunesekere et al., 2006b; Huis in 'T Veld et al., 2011) and $\sigma^{32}$ (Du and Arvidson, 2006; Gunesekere et al., 2006a; Folster et al., 2009) of N. meningitidis. Lastly, the other functional two component systems of $N$. meningitidis, NarQ/NarP (NMB1249/NMB1250) and NtrY/NtrX (NMB0114/NMB0115), have not been found to be involved in the regulation of LPS biosynthesis or oxidative protein folding genes. Microarray analysis of the regulon of the two component system, NarQ/NarP,

\section{REFERENCES}

Abeyrathne, P. D., Daniels, C., Poon, K. K. H., Matewish, M. J., and Lam, J. S. (2005). Functional characterization of waal, a ligase associated with linking o-antigen polysaccharide to the core of Pseudomonas aeruginosa lipopolysaccharide. J. Bacteriol. 187, 3002-3012.

Achard, M. E. S., Hamilton, A. J., Dankowski, T., Heras, B., Schembri, indicates that it regulates genes required for survival in anaerobic conditions in $N$. gonorrhoeae by activating the nitrite reductase, AniA (Lissenden et al., 2000; Overton et al., 2006; Whitehead and Cole, 2006). Although it is known that the NtrY/NtrX two component system, which is involved in nitrogen fixation and metabolism in E. coli (Pawlowski et al., 1991), is down-regulated in response to human whole blood in N. meningitidis (EcheniqueRivera et al., 2011), no transcriptome has been reported.

\section{THE EFFECT OF GENOME REDUCTION ON OM BIOGENESIS}

Overall, the OM biogenesis pathways of LPS biosynthesis and protein oxidation and isomerization remain conserved in function in both Gram-negative species. However, in the commensal E. coli, the LPS pathway is essential to bacterial cell viability due to the loss of OM integrity (Galloway and Raetz, 1990; Onishi et al., 1996), whilst in N. meningitidis, the analogous pathway is not essential (Steeghs et al., 1998). Conversely, the protein oxidation and isomerization pathways which are required for the correct folding of virulence proteins and the protection of proteins in the periplasm from oxidative damage, are not essential in E. coli, but are essential for meningococcal viability (Kumar et al., 2011). Of great interest is the fact that both the endotoxin and protein oxidation/isomerization pathways are co-regulated in such a way as to maintain OM integrity. In E. coli, the number of environmental signals and hence regulatory networks involved in regulating these pathways are very diverse, whereas in the specialized pathogen Neisseria sp., only the regulatory network of the MisR/MisS two component system has been identified so far in performing this dual role. Unlike E. coli MsrABs and thioredoxins which are confined to the cytoplasm, neisserial MsrAB and TlpA are secreted to the outer membrane and periplasm, respectively, are both required for protection from oxidative stress and are regenerated by the protein isomerization pathway (Boschi-Muller et al., 2008). Since Neisseria sp. encounter high levels of ROS and hydrogen peroxide in mucosal secretions during colonization of these surfaces (Criss and Seifert, 2012), the expansion of redox partners for the protein isomerization pathway in this species may suggest that this aspect of the pathway has evolved through niche adaption. Future work to understand the role of redox partners of the protein isomerization pathways in niche adapted pathogens from other genera may uncover other interesting features.

\section{ACKNOWLEDGMENTS}

Susannah Piek and Charlene M. Kahler are supported by the National Health and Medical Research Council (APP572656 and 1003697).
M. S., Edwards, J. L., et al. (2009). A periplasmic thioredoxin-like protein plays a role in defense against oxidative stress in Neisseria gonorrhoeae. Infect. Immun. 77, 4934-4939.

Bader, M., Muse, W., Ballou, D. P., Gassner, C., and Bardwell, J. C. (1999). Oxidative protein folding is driven by the electron transport system. Cell 98, 217-227.
Bader, M. W., Hiniker, A., Regeimbal, J., Goldstone, D., Haebel, P. W., Riemer, J., et al. (2001). Turning a disulfide isomerase into an oxidase: DsbC mutants that imitate DsbA. EMBO J. 20, 1555-1562.

Bader, M. W., Navarre, W. W., Shiau, W., Nikaido, H., Frye, J. G., McClelland, M., et al. (2003). Regulation of Salmonella typhimurium virulence gene expression by cationic antimicrobial peptides. Mol. Microbiol. 50, 219-230.

Bader, M. W., Sanowar, S., Daley, M. E., Schneider, A. R., Cho, U., Xu, W., et al. (2005). Recognition of antimicrobial peptides by a bacterial sensor kinase. Cell 122, 461-472.

Banerjee, A., Wang, R., Uljon, S. N., Rice, P. A., Gotschlich, E. C., and Stein, D. C. (1998). Identification 
of the gene $(\operatorname{lgt} G)$ encoding the lipooligosaccharide beta chain synthesizing glucosyl transferase from Neisseria gonorrhoeae. Proc. Natl. Acad. Sci. U.S.A. 95, 10872-10877.

Bardwell, J. C., Lee, J. O., Jander, G., Martin, N., Belin, D., and Beckwith, J. (1993). A pathway for disulfide bond formation in vivo. Proc. Natl. Acad. Sci. U.S.A. 90, 1038-1042.

Bardwell, J. C., McGovern, K., and Beckwith, J. (1991). Identification of a protein required for disulfide bond formation in vivo. Cell 67, 581-589.

Basler, M., Linhartova, I., Halada, P., Novotna, J., Bezouskova, S., Osicka, R., et al. (2006). The iron-regulated transcriptome and proteome of Neisseria meningitidis serogroup C. Proteomics 6, 6194-6206.

Batchelor, R. A., Haraguchi, G. E., Hull, R. A., and Hull, S. I. (1991). Regulation by a novel protein of the bimodal distribution of lipopolysaccharide in the outer membrane of Escherichia coli. J. Bacteriol. 173, 5699-5704.

Beier, D., and Gross, R. (2006). Regulation of bacterial virulence by two-component systems. Curr. Opin. Microbiol. 9, 143-152.

Belden, W. J., and Miller, S. I. (1994). Further characterization of the PhoP regulon: identification of new PhoP-activated virulence loci. Infect. Immun. 62, 5095-5101.

Berkmen, M., Boyd, D., and Beckwith, J. (2005). The nonconsecutive disulfide bond of Escherichia coli phytase $(\mathrm{AppA})$ renders it dependent on the protein-disulfide isomerase, DsbC. J. Biol. Chem. 280, 11387-11394.

Bessette, P. H., Cotto, J. J., Gilbert, H. F., and Georgiou, G. (1999). In vivo and in vitro function of the Escherichia coli periplasmic cysteine oxidoreductase DsbG. J. Biol. Chem. 274, 7784-7792.

Bishop, R. E., Gibbons, H. S., Guina, T., Trent, M. S., Miller, S. I., and Raetz, C. R. H. (2000). Transfer of palmitate from phospholipids to lipid A in outer membranes of Gram-negative bacteria. EMBO J. 19, 5071-5080.

Bitter, W. (2003). Secretins of Pseudomonas aeruginosa: large holes in the outer membrane. Arch. Microbiol. 179, 307-314.

Bos, M. P., Tefsen, B., Geurtsen, J., and Tommassen, J. (2004). Identification of an outer membrane protein required for the transport of lipopolysaccharide to the bacterial cell surface. Proc. Natl. Acad. Sci. U.S.A. 101, 9417-9422.

Bos, M. P., and Tommassen, J. (2011). The LptD chaperone LptE is not directly involved in lipopolysaccharide transport in Neisseria meningitidis. J. Biol. Chem. 286, 28688-28696.

Boschi-Muller, S., Gand, A., and Branlant, G. (2008). The methionine sulfoxide reductases: catalysis and substrate specificities. Arch. Biochem. Biophys. 474, 266-273.

Bouwman, C. W., Kohli, M., Killoran, A., Touchie, G. A., Kadner, R. J., and Martin, N. L. (2003). Characterization of SrgA, a Salmonella enterica serovar Typhimurium virulence plasmidencoded paralogue of the disulfide oxidoreductase DsbA, essential for biogenesis of plasmid-encoded fimbriae. J. Bacteriol. 185, 991-1000.

Braun, M., and Silhavy, T. J. (2002). Imp/OstA is required for cell envelope biogenesis in Escherichia coli. Mol. Microbiol. 45, 1289-1302.

Bronner, D., Clarke, R., and Whitfield, C. (1994). Identification of an ATP-binding cassette transport system required for translocation of lipopolysaccharide $\mathrm{O}$-antigen side-chains across the cytoplasmic membrane of Klebsiella pneumoniae serotype O1. Mol. Microbiol. 14, 505-519.

Brot, N., Collet, J.-F., Johnson, L. C., Jönsson, T. J., Weissbach, H., and Lowther, W. T. (2006). The thioredoxin domain of Neisseria gonorrhoeae PilB can use electrons from DsbD to reduce downstream methionine sulfoxide reductases. J. Biol. Chem. 281, 32668-32675.

Carty, S. M., Sreekumar, K. R., and Raetz, C. R. H. (1999). Effect of cold shock on lipid A biosynthesis in Escherichia coli. J. Biol. Chem. 274, 9677-9685.

Chen, J., Song, J., Zhang, S., Wang, Y., Cui, D., and Wang, C. (1999). Chaperone activity of DsbC. J. Biol. Chem. 274, 19601-19605.

Chng, S.-S., Gronenberg, L. S., and Kahne, D. (2010a). Proteins required for lipopolysaccharide assembly in Escherichia coli form a transenvelope complex. Biochemistry 49, 4565-4567.

Chng, S. S., Ruiz, N., Chimalakonda, G., Silhavy, T. J., and Kahne, D. (2010b). Characterization of the two-protein complex in Escherichia coli responsible for lipopolysaccharide assembly at the outer membrane. Proc. Natl. Acad. Sci. U.S.A. 107, 5363-5368.

Cho, S.-H., and Beckwith, J. (2009). Two snapshots of electron transport across the membrane. J. Biol. Chem. 284, 11416-11424.

Cho, S.-H., Porat, A., Ye, J., and Beckwith, J. (2007). Redox-active cysteines of a membrane electron transporter DsbD show dual compartment accessibility. EMBO J. 26, 3509-3520.

Collet, J.-F., Riemer, J., Bader, M W., and Bardwell, J. C. A. (2002). Reconstitution of a disulfide isomerization system. J. Biol. Chem. 277, 26886-26892.

Cornelis, G. R. (2006). The type III secretion injectisome. Nat. Rev. Microbiol. 4, 811-825.

Costanzo, A., and Ades, S. E. (2006) Growth phase-dependent regulation of the extracytoplasmic stress factor, sigmaE, by guanosine $3^{\prime}$, $5^{\prime}$-bispyrophosphate (ppGpp). J. Bacteriol. 188, 4627-4634.

Cox, A. D., Wright, J. C., Li, J., Hood, D. W., Moxon, E. R., and Richards, J. C. (2003). Phosphorylation of the lipid A region of meningococcal lipopolysaccharide: identification of a family of transferases that add phosphoethanolamine to lipopolysaccharide. J. Bacteriol. 185 3270-3277.

Creeger, E. S., and Rothfield, L. I. (1979). Cloning of genes for bacterial glycosyltransferases. I. Selection of hybrid plasmids carrying genes for two glucosyltransferases. J. Biol. Chem. 254, 804-810.

Criss, A. K., and Seifert, H. S. (2012). A bacterial siren song: intimate interactions between Neisseria and neutrophils. Nat. Rev. Microbiol. 10, 178-190.

Cullen, T. W., and Trent, M. S. (2010). A link between the assembly of flagella and lipooligosaccharide of the Gram-negative bacterium Campylobacter jejuni. Proc. Natl. Acad. Sci. U.S.A. 107, 5160-5165.

Dailey, F., and Berg, H. (1993). Mutants in disulfide bond formation that disrupt falgellar assembly in Escherichia coli. Proc. Natl. Acad. Sci. U.S.A. 90, 1043-1047.

Danese, P. N., and Silhavy, T. J. (1997). The sigma(E) and the Cpx signal transduction systems control the synthesis of periplasmic proteinfolding enzymes in Escherichia coli. Genes Dev. 11, 1183-1193.

Danese, P. N., and Silhavy, T. J. (1998) CpxP, a stress-combative member of the Cpx regulon. J. Bacteriol. 180, 831-839.

Danese, P. N., Snyder, W. B., Cosma, C. L., Davis, L. J., and Silhavy, T. J. (1995). The Cpx two-component signal transduction pathway of Escherichia coli regulates transcription of the gene specifying the stress-inducible periplasmic protease, DegP. Genes Dev. 9, 387-398.

Darby, N. J., Raina, S., and Creighton, T. E. (1998). Contributions of substrate binding to the catalytic activity of DsbC. Biochemistry 37, 783-791.

Denoncin, K., Vertommen, D., Paek, E. and Collet, J. F. (2010). The proteindisulfide isomerase DsbC cooperates with SurA and DsbA in the assembly of the essential beta-barrel protein LptD. J. Biol. Chem. 285, 29425-29433.

Depuydt, M., Leonard, S. E. Vertommen, D., Denoncin, K., Morsomme, P., Wahni, K., et al. (2009). A periplasmic reducing system protects single cysteine residues from oxidation. Science 326, 1109-1111.

De Wulf, P., McGuire, A. M., Liu, X., and Lin, E. C. C. (2002). Genome-wide profiling of promoter recognition by the two-component response regulator CpxR-P in Escherichia coli. J. Biol. Chem. 277, 26652-26661.

Dietrich, G., Kurz, S., Hubner, C., Aepinus, C., Theiss, S., Guckenberger, M., et al. (2003). Transcriptome analysis of Neisseria meningitidis during infection. J. Bacteriol. 185, 155-164.

Doerrler, W. T., Reedy, M. C., and Raetz, C. R. (2001). An Escherichia coli mutant defective in lipid export. J. Biol. Chem. 276, 11461-11464.

Du, Y., and Arvidson, C. G. (2006). $\mathrm{RpoH}$ mediates the expression of some, but not all, genes induced in Neisseria gonorrhoeae adherent to epithelial cells. Infect. Immun. 74, 2767-2776.

Dutton, R. J., Boyd, D., Berkmen, M., and Beckwith, J. (2008). Bacterial species exhibit diversity in their mechanisms and capacity for protein disulfide bond formation. Proc. Natl. Acad. Sci. U.S.A. 105 11933-11938.

Echenique-Rivera, H., Muzzi, A., Del Tordello, E., Seib, K. L., Francois, P., Rappuoli, R., et al. (2011). Transcriptome analysis of Neisseria meningitidis in human whole blood and mutagenesis studies identify virulence factors involved in blood survival. PLoS Pathog. 7:e1002027. doi: 10.1371/journal.ppat.1002027

Feldman, M. F., Marolda, C. L., Monteiro, M. A., Perry, M. B. Parodi, A. J., and Valvano, M. A. (1999). The activity of a putative polyisoprenol-linked sugar translocase (Wzx) involved in Escherichia coli $\mathrm{O}$ antigen assembly is independent of the chemical structure of the O repeat. J. Biol. Chem. 274, 35129-35138.

Figueroa-Bossi, N., Lemire, S., Maloriol, D., Balbontín, R. Casadesús, J., and Bossi, L. 
(2006). Loss of $\mathrm{Hfq}$ activates the $\sigma^{\mathrm{E}}$-dependent envelope stress response in Salmonella enterica. Mol. Microbiol. 62, 838-852.

Folster, J. P., Johnson, P. J., Jackson, L., Dhulipali, V., Dyer, D. W., and Shafer, W. M. (2009). MtrR modulates $r p o H$ expression and levels of antimicrobial resistance in Neisseria gonorrhoeae. J. Bacteriol. 191, 287-297.

Frirdich, E., Lindner, B., Holst, O., and Whitfield, C. (2003). Overexpression of the waaZ gene leads to modification of the structure of the inner core region of Escherichia coli lipopolysaccharide, truncation of the outer core, and reduction of the amount of $\mathrm{O}$ polysaccharide on the cell surface. J. Bacteriol. 185, 1659-1671.

Galan, J. E., and Wolf-Watz, H. (2006). Protein delivery into eukaryotic cells by type III secretion machines. Nature 444, 567-573.

Galanos, C., Lüderitz, O., Rietschel, E. T., Westphal, O., Brade, H., Brade, L., et al. (1985). Synthetic and natural Escherichia coli free lipid A express identical endotoxic activities. Eur. J. Biochem. 148, 1-5.

Galloway, S. M., and Raetz, C. R. (1990). A mutant of Escherichia coli defective in the first step of endotoxin biosynthesis. J. Biol. Chem. 265, 6394-6402.

Galperin, M. Y., and Jedrzejas, M. J. (2001). Conserved core structure and active site residues in alkaline phosphatase superfamily enzymes. Proteins 45, 318-324.

Garcia Vescovi, E., Soncini, F. C., and Groisman, E. A. (1996). Mg2+ as an extracellular signal: environmental regulation of Salmonella virulence. Cell 84, 165-174.

Georgiades, K. (2012). Genomics of epidemic pathogens. Clin. Microbiol. Infect. 18, 213-217.

Gibbons, H. S., Kalb, S. R., Cotter, R. J., and Raetz, C. R. H. (2005). Role of $\mathrm{Mg} 2+$ and $\mathrm{pH}$ in the modification of Salmonella lipid A after endocytosis by macrophage tumour cells. Mol. Microbiol. 55, 425-440.

Gibbons, H. S., Lin, S., Cotter, R. J., and Raetz, C. R. H. (2000). Oxygen requirement for the biosynthesis of the S-2-hydroxymyristate moiety in Salmonella typhimurium lipid A. J. Biol. Chem. 275, 32940-32949.

Gilbert, M., Watson, D. C., Cunningham, A. M., Jennings, M. P., Young, N. M., and Wakarchuk, W. W. (1996). Cloning of the lipooligosaccharide alpha-2, 3-sialyltransferase from the bacterial pathogens Neisseria meningitidis and Neisseria gonorrhoeae. J. Biol. Chem. 271, 28271-28276.

Grifantini, R., Bartolini, E., Muzzi, A., Draghi, M., Frigimelica, E., Berger, J., et al. (2002a). Gene expression profile in Neisseria meningitidis and Neisseria lactamica upon host-cell contact: from basic research to vaccine development. Ann. N.Y. Acad. Sci. 975, 202-216.

Grifantini, R., Bartolini, E., Muzzi, A., Draghi, M., Frigimelica, E., Berger, J., et al. (2002b). Previously unrecognized vaccine candidates against group B meningococcus identified by DNA microarrays. Nat. Biotechnol. 20, 914-921.

Grifantini, R., Sebastian, S., Frigimelica, E., Draghi, M. Bartolini, E., Muzzi, A., et al. (2003). Identification of iron-activated and -repressed Fur-dependent genes by transcriptome analysis of Neisseria meningitidis group B. Proc. Natl. Acad. Sci. U.S.A. 100, 9542-9547.

Grimshaw, J. P. A., Stirnimann, C. U., Brozzo, M. S., Malojcic, G., Grütter, M. G., Capitani, G., et al. (2008). DsbL and DsbI form a specific dithiol oxidase system for periplasmic arylsulfate sulfotransferase in uropathogenic Escherichia coli. J. Mol. Biol. 380, 667-680.

Gronow, S., Brabetz, W., and Brade, H. (2000). Comparative functional characterization in vitro of heptosyltransferase I (WaaC) and II (WaaF) from Escherichia coli. Eur. J. Biochem. 267, 6602-6611.

Guckenberger, M., Kurz, S., Aepinus, C., Theiss, S., Haller, S., Leimbach, T., et al. (2002). Analysis of the heat shock response of Neisseria meningitidis with CDNA- and oligonucleotide-based DNA microarrays. J. Bacteriol. 184, 2546-2551.

Gunesekere, I. C., Kahler, C. M., Powell, D. R., Snyder, L. A., Saunders, N. J., Rood, J. I., et al. (2006a). Comparison of the RpoH-dependent regulon and general stress response in Neisseria gonorrhoeae. J. Bacteriol. 188, 4769-4776.

Gunesekere, I. C., Kahler, C. M., Ryan, C. S., Snyder, L. A., Saunders, N. J., Rood, J. I., et al. (2006b). Ecf, an alternative sigma factor from Neisseria gonorrhoeae, controls expression of $m s r A B$, which encodes methionine sulfoxide reductase. J. Bacteriol. 188, 3463-3469.

Gunn, J. S., Lim, K. B., Krueger, J., Kim, K., Guo, L., Hackett, M., et al. (1998). PmrA-PmrBregulated genes necessary for 4-aminoarabinose lipid A modification and polymyxin resistance. Mol. Microbiol. 27, 1171-1182.

Guo, L., Lim, K. B., Gunn, J. S., Bainbridge, B., Darveau, R P., Hackett, M., et al. (1997). Regulation of lipid A modifications by Salmonella typhimurium virulence genes phoP-phoQ. Science 276, 250-253.

Guo, L., Lim, K. B., Poduje, C. M., Daniel, M., Gunn, J. S., Hackett, M., et al. (1998). Lipid A acylation and bacterial resistance against vertebrate antimicrobial peptides. Cell 95, 189-198.

Haebel, P., Goldstone, D., Katzen, F., Beckwith, J., and Metcalf, P. (2002). The disulfide bond isomerase DsbC is activated by an immunoglobulinfold thiol oxidoreductase: crystal structure of the DsbCDsbDalpha complex. EMBO J. 21, 4774-4784.

Harvey, H. A., Swords, W. E., and Apicella, M. A. (2001). The mimicry of human glycolipids and glycosphingolipids by the lipooligosaccharides of pathogenic Neisseria and Haemophilus. J. Autoimmun. 16, 257-262.

Heinrichs, D. E., Yethon, J. A., Amor, P. A., and Whitfield, C. (1998a). The assembly system for the outer core portion of R1- and R4-type lipopolysaccharides of Escherichia coli. J. Biol. Chem. 273, 29497-29505.

Heinrichs, D. E., Yethon, J. A., and Whitfield, C. (1998b). Molecular basis for structural diversity in the core regions of the lipopolysaccharides of Escherichia coli and Salmonella enterica. Mol. Microbiol. 30, 221-232.

Helander, M., Kilpeläinen, I., and Vaara, M. (1994). Increased substitution of phosphate groups in lipopolysaccharides and lipid A of the polymyxin-resistant pmrA mutants of Salmonella typhimurium: a 31P-NMR study. Mol. Microbiol. 11, 481-487.

Heras, B., Edeling, M. A., Schirra, H. J., Raina, S., and Martin, J. L. (2004). Crystal structures of the DsbG disulfide isomerase reveal an unstable disulfide. Proc. Natl. Acad. Sci. U.S.A. 101, 8876-8881.

Heras, B., Shouldice, S. R., Totsika, M., Scanlon, M. J., Schembri, M. A., and Martin, J. L. (2009). DSB proteins and bacterial pathogenicity. Nat. Rev. Microbiol. 7, 215-225.

Herrera, C. M., Hankins, J. V., and Trent, M. S. (2010). Activation of PmrA inhibits LpxT-dependent phosphorylation of lipid A promoting resistance to antimicrobial peptides. Mol. Microbiol. 76, 1444-1460.

Hiniker, A., and Bardwell, J. C. (2004). In vivo substrate specificity of periplasmic disulfide oxidoreductases. J. Biol. Chem. 279, 12967-12973.

Hizukuri, Y., Yakushi, T., Kawagishi, I., and Homma, M. (2006). Role of the intramolecular disulfide bond in FlgI, the flagellar P-ring component of Escherichia coli. J. Bacteriol. 188, 4190-4197.

Horne, J., D'auvergne, E. J., Coles, M., Velkov, T., Chin, Y., Charman, W. N., et al. (2007). Probing the flexibility of the DsbA oxidoreductase from Vibrio cholerae-a 15N $1 \mathrm{H}$ heteronuclear NMR relaxation analysis of oxidized and reduced forms of DsbA. J. Mol. Biol. 371, 703-716.

Huber-Wunderlich, M., and Glockshuber, R. (1998). A single dipeptide sequence modulates the redox properties of a whole enzyme family. Fold. Des. 3, 161-171.

Huis in 'T Veld, R. A., Willemsen, A. M., Van Kampen, A. H., Bradley, E. J., Baas, F., Pannekoek, Y., et al. (2011). Deep sequencing whole transcriptome exploration of the sigmaE regulon in Neisseria meningitidis. PLoS ONE 6:e29002. doi: 10.1371/journal.pone.0029002

Hultgren, S. J., Abraham, S., Caparon, M., Falk, P., St. Geme, J. W. 3rd., and Normark, S. (1993). Pilus and nonpilus bacterial adhesins: assembly and function in cell recognition. Cell 73, 887-901.

Hultgren, S. J., Normark, S., and Abraham, S. N. (1991). Chaperoneassisted assembly and molecular architecture of adhesive pili. Annu. Rev. Microbiol. 45, 383-415.

Inaba, K., and Ito, K. (2008). Structure and mechanisms of the DsbBDsbA disulfide bond generation machine. Biochim. Biophys. Acta. 1783, 520-529.

Inaba, K., Murakami, S., Nakagawa, A., Iida, H., Kinjo, M., Ito, K., et al. (2009). Dynamic nature of disulphide bond formation catalysts revealed by crystal structures of DsbB. EMBO J. 28, 779-791.

Inaba, K., Murakami, S., Suzuki, M., Nakagawa, A., Yamashita, E., Okada, K., et al. (2006a). Crystal structure of the DsbB-DsbA complex reveals a mechanism of disulfide bond generation. Cell 127, 789-801.

Inaba, K., Takahashi, Y. H., Ito, K., and Hayashi, S. (2006b). Critical role of a thiolate-quinone charge transfer complex and its adduct form in de novo disulfide bond generation by DsbB. Proc. Natl. Acad. Sci. U.S.A. 103, 287-292. 
Inaba, K., Takahashi, Y.-H., and Ito, K. (2004). DsbB Elicits a red-shift of bound ubiquinone during the catalysis of DsbA oxidation. J. Biol. Chem. 279, 6761-6768.

Inaba, K., Takahashi, Y. H., and Ito, K. (2005). Reactivities of quinone-free DsbB from Escherichia coli. J. Biol. Chem. 280, 33035-33044.

Jacob-Dubuisson, F., Pinkner, J., Xu, Z., Striker, R., Padmanhaban, A., and Hultgren, S. J. (1994). PapD chaperone function in pilus biogenesis depends on oxidant and chaperone-like activities of DsbA. Proc. Natl. Acad. Sci. U.S.A. 91, 11552-11556.

Jamet, A., Rousseau, C., Monfort, J. B., Frapy, E., Nassif, X., and Martin, P. (2009). A two-component system is required for colonization of host cells by meningococcus. Microbiology 155, 2288-2295.

Jander, G., Martin, N. L., and Beckwith, J. (1994). Two cysteines in each periplasmic domain of the membrane protein DsbB are required for its function in protein disulfide bond formation. $E M B O \mathrm{~J} .13$, 5121-5127.

Jennings, H. J., Bhattacharjee, A. K., Kenne, L., Kenny, C. P., and Calver, G. (1980). The R-type lipopolysaccharides of Neisseria meningitidis. Can. J. Biochem. 58, 128-136.

Jennings, M. P., Hood, D. W., Peak, I. R., Virji, M., and Moxon, E. R. (1995). Molecular analysis of a locus for the biosynthesis and phase-variable expression of the lacto-N-neotetraose terminal lipopolysaccharide structure in Neisseria meningitidis. Mol. Microbiol. 18, 729-740.

Jennings, M. P., Srikhanta, Y. N., Moxon, E. R., Kramer, M., Poolman, J. T., Kuipers, B., et al. (1999). The genetic basis of the phase variation repertoire of lipopolysaccharide immunotypes in Neisseria meningitidis. Microbiology 145( $\mathrm{Pt}$ 11), 3013-3021.

Johnson, C. R., Newcombe, J., Thorne, S., Borde, H. A., Eales-Reynolds, L. J., Gorringe, A. R., et al. (2001). Generation and characterization of a PhoP homologue mutant of Neisseria meningitidis. Mol. Microbiol. 39, 1345-1355.

Joly, J. C., and Swartz, J. R. (1997). In vitro and in vivo redox states of the Escherichia coli periplasmic oxidoreductases DsbA and DsbC. Biochemistry 36, 10067-10072.

Jones, C. H., Danese, P. N., Pinkner, J. S., Silhavy, T. J., and Hultgren, S. J. (1997). The chaperone-assisted membrane release and folding pathway is sensed by two signal transduction systems. EMBO J. 16, 6394-6406.

Kadokura, H., and Beckwith, J. (2002). Four cysteines of the membrane protein DsbB act in concert to oxidise its substrate DsbA. EMBO J. 21, 2354-2363.

Kadokura, H., and Beckwith, J. (2010). Mechanisms of oxidative protein folding in the bacterial cell envelope. Antioxid. Redox. Signal. 13, 1231-1246.

Kadokura, H., Bader, M., Tian, H., Bardwell, J. C., and Beckwith, J. (2000). Roles of a conserved arginine residue of DsbB in linking protein disulfide-bond-formation pathway to the respiratory chain of Escherichia coli. Proc. Natl. Acad. Sci. U.S.A. 97, 10884-10889.

Kadokura, H., Tian, H., Zander, T., Bardwell, J., and Beckwith, J. (2004). Snapshots of DsbA in action: detection of proteins in the process of oxidative folding. Science 303, 534-537.

Kahler, C., and Stephens, D. (1998). Genetic basis for biosynthesis, structure, and function of meningococcal lipooligosaccharide (endotoxin) Crit. Rev. Microbiol. 24 281-334.

Kahler, C. M., Carlson, R. W., Rahman, M. M., Martin, L. E., and Stephens, D. S. (1996a). Inner core biosynthesis of lipooligosaccharide (LOS) in Neisseria meningitidis serogroup B: identification and role in LOS assembly of the alpha1, 2 $\mathrm{N}$-acetylglucosamine transferase (RfaK). J. Bacteriol. 178, 1265-1273.

Kahler, C. M., Carlson, R. W., Rahman, M. M., Martin, L. E., and Stephens, D. S. (1996b). Two glycosyltransferase genes, lgtF and $r f a K$, constitute the lipooligosaccharide ice (inner core extension) biosynthesis operon of Neisseria meningitidis. J. Bacteriol. 178, 6677-6684.

Kahler, C. M., Datta, A., Tzeng, Y. L., Carlson, R. W., and Stephens, D. S. (2005). Inner core assembly and structure of the lipooligosaccharide of Neisseria meningitidis: capacity of strain NMB to express all known immunotype epitopes. Glycobiology 15, 409-419.

Kahler, C. M., Lyons-Schindler, S., Choudhury, B., Glushka, J., Carlson, R. W., and Stephens, D. S. (2006). O-Acetylation of the terminal $\mathrm{N}$-acetylglucosamine of the lipooligosaccharide inner core in Neisseria meningitidis. Influence on inner core structure and assembly. J. Biol. Chem. 281, 19939-19948.

Kahler, C. M., Martin, L. E., Shih, G. C., Rahman, M. M., Carlson, R. W., and Stephens, D. S. (1998).
The (alpha2->8)-linked polysialic acid capsule and lipooligosaccharide structure both contribute to the ability of serogroup B Neisseria meningitidis to resist the bactericidal activity of normal human serum. Infect. Immun. 66, 5939-5947.

Kanipes, M. I., Lin, S., Cotter, R. J., and Raetz, C. R. H. (2001). Ca2+induced phosphoethanolamine transfer to the outer 3-deoxy-dmanno-octulosonic acid moiety of Escherichia coli lipopolysaccharide. J. Biol. Chem. 276, 1156-1163.

Karow, M., and Georgopoulos, C. (1993). The essential Escherichia coli $m s b A$ gene, a multicopy suppressor of null mutations in the htrB gene, is related to the universally conserved family of ATP-dependent translocators. Mol. Microbiol. 7 , 69-79.

Kato, A., and Groisman, E. A. (2004) Connecting two-component regulatory systems by a protein that protects a response regulator from dephosphorylation by its cognate sensor. Genes Dev. 18, 2302-2313.

Katzen, F., and Beckwith, J. (2000) Transmembrane electron transfer by the membrane protein $\mathrm{DsbD}$ occurs via a disulfide bond cascade. Cell 103, 769-779.

Kawasaki, K., Ernst, R. K., and Miller, S. I. (2004a). 3-O-Deacylation of lipid A by PagL, a PhoP/PhoQregulated deacylase of Salmonella typhimurium, modulates signaling through Toll-like receptor 4. J. Biol. Chem. 279, 20044-20048.

Kawasaki, K., Ernst, R. K., and Miller, S. I. (2004b). Deacylation and palmitoylation of lipid A by Salmonellae outer membrane enzymes modulate host signaling through Toll-like receptor 4. J. Endotoxin. Res. 10, 439-444.

Kido, N., Torgov, V. I., Sugiyama, T., Uchiya, K., Sugihara, H., Komatsu, T., et al. (1995). Expression of the O9 polysaccharide of Escherichia coli: sequencing of the E. coli OS $\mathrm{rfb}$ gene cluster, characterization of mannosyl transferases, and evidence for an ATP-binding cassette transport system. J. Bacteriol. 177, 2178-2187.

Klee, S. R., Nassif, X., Kusecek, B., Merker, P., Beretti, J. L., Achtman, M., et al. (2000). Molecular and biological analysis of eight genetic islands that distinguish Neisseria meningitidis from the closely related pathogen Neisseria gonorrhoeae. Infect. Immun. 68, 2082-2095.

Kobayashi, T., and Ito, K. (1999). Respiratory chain strongly oxidizes the CXXC motif of DsbB in the Escherichia coli disulfide bond formation pathway. EMBO J. 18, 1192-1198.

Kobayashi, T., Takahashi, Y., and Ito, K. (2001). Identification of a segment of DsbB essential for its respirationcoupled oxidation. Mol. Microbiol. 39, 158-165.

Kox, L. F., Wosten, M. M., and Groisman, E. A. (2000). A small protein that mediates the activation of a two-component system by another two-component system. EMBO J. 19, 1861-1872.

Kulshin, V. A., Zahringer, U., Lindner, B., Frasch, C. E., Tsai, C. M., Dmitriev, B. A., et al. (1992). Structural characterization of the lipid A component of pathogenic Neisseria meningitidis. J. Bacteriol. 174, 1793-1800.

Kulshin, V. A., Zähringer, U., Lindner, B., Jäger, K.-E., Dmitriev, B. A., and Rietschel, E. T. (1991). Structural characterization of the lipid A component of Pseudomonas aeruginosa wild-type and rough mutant lipopolysaccharides. Eur. J. Biochem. 198, 697-704

Kumar, P., Sannigrahi, S., Scoullar, J., Kahler, C. M., and Tzeng, Y.-L. (2011). Characterization of DsbD in Neisseria meningitidis. Mol. Microbiol. 79, 1557-1573.

Kurz, S., Hubner, C., Aepinus, C., Theiss, S., Guckenberger, M., Panzner, U., et al. (2003). Transcriptome-based antigen identification for Neisseria meningitidis. Vaccine 21, 768-775.

Lafaye, C., Iwema, T., Carpentier, P., Jullian-Binard, C., Kroll, J. S., Collet, J.-F., et al. (2009). Biochemical and structural study of the homologues of the thiol-disulfide oxidoreductase DsbA in Neisseria meningitidis. J. Mol. Biol. 392, 952-966.

Lee, H., Hsu, F.-F., Turk, J., and Groisman, E. A. (2004). The PmrAregulated $p m r C$ gene mediates phosphoethanolamine modification of lipid A and polymyxin resistance in Salmonella enterica. J. Bacteriol. 186, 4124-4133.

Lee, J. H., Lee, K. L., Yeo, W. S., Park, S. J., and Roe, J. H. (2009). SoxRSmediated lipopolysaccharide modification enhances resistance against multiple drugs in Escherichia coli. J. Bacteriol. 191, 4441-4450.

Lewis, L. A., Choudhury, B., Balthazar, J. T., Martin, L. E., Ram, S., Rice, P. A., et al. (2009). Phosphoethanolamine substitution of lipid A and resistance of Neisseria gonorrhoeae to cationic antimicrobial peptides and complement-mediated killing by normal human serum. Infect. Immun. 77, 1112-1120. 
Liechti, G., and Goldberg, J. B. (2012). Outer membrane biogenesis in Escherichia coli, Neisseria meningitidis, and Helicobacter pylori: paradigm deviations in $H$. pylori. Front. Cell. Infect. Microbiol. 2:29. doi: 10.3389/fcimb.2012.00029

Lin, D., Kim, B., and Slauch, J. M. (2009). DsbL and DsbI contribute to periplasmic disulfide bond formation in Salmonella enterica serovar Typhimurium. Microbiology 155, 4014-4024.

Lissenden, S., Mohan, S., Overton, T., Regan, T., Crooke, H., Cardinale, J. A., et al. (2000). Identification of transcription activators that regulate gonococcal adaptation from aerobic to anaerobic or oxygenlimited growth. Mol. Microbiol. 37, 839-855.

Liu, D., Cole, R. A., and Reeves, P. R. (1996). An O-antigen processing function for Wzx (RfbX): a promising candidate for O-unit flippase. J. Bacteriol. 178, 2102-2107.

Liu, M., John, C. M., and Jarvis, G. A. (2010). Phosphoryl moieties of lipid A from Neisseria meningitidis and N. gonorrhoeae lipooligosaccharides play an important role in activation of both MyD88- and TRIF-dependent TLR4-MD-2 signaling pathways. J. Immunol. 185, 6974-6984.

Loppnow, H., Brade, L., Brade, H., Rietschel, E. T., Kusumoto, S., Shiba, T., et al. (1986). Induction of human interleukin 1 by bacterial and synthetic lipid A. Eur. J. Immunol. 16, 1263-1267.

Mackinnon, F. G., Borrow, R., Gorringe, A. R., Fox, A. J., Jones, D. M., and Robinson, A. (1993). Demonstration of lipooligosaccharide immunotype and capsule as virulence factors for Neisseria meningitidis using an infant mouse intranasal infection model. Microb. Pathog. 15, 359-366.

Mackinnon, F. G., Cox, A. D., Plested, J. S., Tang, C. M., Makepeace, K., Coull, P. A., et al. (2002). Identification of a gene (lpt3) required for the addition of phosphoethanolamine to the lipopolysaccharide inner core of Neisseria meningitidis and its role in mediating susceptibility to bactericidal killing and opsonophagocytosis. Mol. Microbiol. 43, 931-943.

Mandrell, R. E., Kim, J. J., John, C. M., Gibson, B. W., Sugai, J. V., Apicella, M. A., et al. (1991). Endogenous sialylation of the lipooligosaccharides of Neisseria meningitidis. J. Bacteriol. 173, 2823-2832.
Marolda, C. L., Tatar, L. D., Alaimo, C., Aebi, M., and Valvano, M. A. (2006) Interplay of the Wzx translocase and the corresponding polymerase and chain length regulator proteins in the translocation and periplasmic assembly of lipopolysaccharide $\mathrm{O}$ antigen. J. Bacteriol. 188, 5124-5135.

Martin, J. (1995). Thioredoxin-a fold for all reasons. Structure 3, 245-250.

Martin, J., Bardwell, J., and Kuriyan, J. (1993a). Crystal structure of the DsbA protein required for disulfide bond formation in vivo. Nature 365 , 464-468.

Martin, J. L., Waksman, G., Bardwell, J. C., Beckwith, J., and Kuriyan, J. (1993b). Crystallization of DsbA, an Escherichia coli protein required for disulphide bond formation in vivo. J. Mol. Biol. 230, 1097-1100.

Mascher, T., Helmann, J. D., and Unden, G. (2006). Stimulus perception in bacterial signaltransducing histidine kinases. Microbiol. Mol. Biol. Rev. 70, 910-938.

McCarthy, A. A., Haebel, P. W., Torronen, A., Rybin, V., Baker, E. N., and Metcalf, P. (2000). Crystal structure of the protein disulfide bond isomerase, DsbC, from Escherichia coli. Nat. Struct. Mol. Biol. 7, 196-199.

Mecsas, J., Rouviere, P. E., Erickson, J. W., Donohue, T. J., and Gross, C. A. (1993). The activity of sigma E, an Escherichia coli heat-inducible sigma-factor, is modulated by expression of outer membrane proteins. Genes Dev. 7, 2618-2628.

Merhej, V., Royer-Carenzi, M., Pontarotti, P., and Raoult, D. (2009). Massive comparative genomic analysis reveals convergent evolution of specialized bacteria. Biol. Direct. 4:13. doi: 10.1186/1745-6150-4-13

Miki, T., Okada, N., and Danbara, H. (2004). Two periplasmic disulfide oxidoreductases, DsbA and SrgA, target outer membrane protein SpiA, a component of the Salmonella pathogenicity island 2 typpe III secretion system. J. Biol. Chem. 279, 34631-34642.

Miki, T., Okada, N., Kim, Y., Abe, A. and Danbara, H. (2008). DsbA directs efficient expression of outer membrane secretin EscC of the enteropathogenic Escherichia coli type III secretion apparatus. Microb. Pathog. 44, 151-158.

Mileykovskaya, E., and Dowhan, W. (1997). The Cpx two-component signal transduction pathway is activated in Escherichia coli mutant strains lacking phosphatidylethanolamine. J. Bacteriol. 179, 1029-1034.

Missiakas, D., Betton, J. M., and Raina, S. (1996). New components of protein folding in extracytoplasmic compartments of Escherichia coli SurA, FkpA and Skp/OmpH. Mol. Microbiol. 21, 871-884.

Moon, K., and Gottesman, S. (2009). A PhoQ/P-regulated small RNA regulates sensitivity of Escherichia coli to antimicrobial peptides. Mol. Microbiol. 74, 1314-1330.

Moran, A. P., Prendergast, M. M., and Appelmelk, B. J. (1996). Molecular mimicry of host structures by bacterial lipopolysaccharides and its contribution to disease. FEMS Immunol. Med. Microbiol. 16, 105-115.

Moran, E. E., Brandt, B. L., and Zollinger, W. D. (1994). Expression of the L8 lipopolysaccharide determinant increases the sensitivity of Neisseria meningitidis to serum bactericidal activity. Infect. Immun. 62, 5290-5295.

Naessan, C. L., Egge-Jacobsen, W. Heiniger, R. W., Wolfgang, M. C. Aas, F. E., Rohr, A., et al. (2008) Genetic and functional analyses of PptA, a phospho-form transferase targeting type IV pili in Neisseria gonorrhoeae. J. Bacteriol. 190, 387-400.

Nakayama, S., and Watanabe, $\mathrm{H}$. (1995). Involvement of CpxA, a sensor of a two-component regulatory system, in the $\mathrm{pH}$-dependent regulation of expression of Shigella sonnei virF gene. J. Bacteriol. 177 , 5062-5069.

Narita, S.-I., and Tokuda, H. (2009). Biochemical characterization of an $\mathrm{ABC}$ transporter LptBFGC complex required for the outer membrane sorting of lipopolysaccharides. FEBS Lett. 583, 2160-2164.

Nelson, J., and Creighton, T. (1994). Reactivity and ionization of the active site cysteine residues of DsbA a protein required for disulfide bond formation in vivo. Biochemistry 33 5974-5983.

Newcombe, J., Eales-Reynolds, L. J., Wootton, L., Gorringe, A. R., Funnell, S. G., Taylor, S. C., et al. (2004). Infection with an avirulent phoP mutant of Neisseria meningitidis confers broad cross-reactive immunity. Infect. Immun. 72, 338-344.

Newcombe, J., Jeynes, J. C., Mendoza, E., Hinds, J., Marsden, G. L., Stabler, R. A., et al. (2005). Phenotypic and transcriptional characterization of the meningococcal PhoPQ system, a magnesium-sensing two-component regulatory system that controls genes involved in remodeling the meningococcal cell surface. J. Bacteriol. 187, 4967-4975.

Onishi, H. R., Pelak, B. A., Gerckens, L. S., Silver, L. L., Kahan, F. M., Chen, M. H., et al. (1996). Antibacterial agents that inhibit lipid A biosynthesis. Science 274, 980-982.

Oshima, T., Aiba, H., Masuda, Y., Kanaya, S., Sugiura, M., Wanner, B. L., et al. (2002). Transcriptome analysis of all two-component regulatory system mutants of Escherichia coli K-12. Mol. Microbiol. 46, 281-291.

Otto, K., and Silhavy, T. J. (2002). Surface sensing and adhesion of Escherichia coli controlled by the Cpx-signaling pathway. Proc. Natl. Acad. Sci. U.S.A. 99, 2287-2292.

Overgaard, M., Kallipolitis, B., and Valentin-Hansen, P. (2009). Modulating the bacterial surface with small RNAs: a new twist on PhoP/Q-mediated lipopolysaccharide modification. Mol. Microbiol. 74, 1289-1294.

Overton, T. W., Whitehead, R., Li, Y., Snyder, L. A., Saunders, N. J., Smith, H., et al. (2006). Coordinated regulation of the Neisseria gonorrhoeae truncated denitrification pathway by the nitric oxide-sensitive repressor, NsrR, and nitrite-insensitive NarQ-NarP. J. Biol. Chem. 281, 33115-33126.

Papenfort, K., Pfeiffer, V., Mika, F, Lucchini, S., Hinton, J. C., and Vogel, J. (2006). SigmaE-dependent small RNAs of Salmonella respond to membrane stress by accelerating global omp mRNA decay. Mol Microbiol. 62, 1674-1688.

Parkhill, J., Achtman, M., James, K. D., Bentley, S. D., Churcher, C., Klee, S. R., et al. (2000). Complete DNA sequence of a serogroup A strain of Neisseria meningitidis Z2491. Nature 404, 502-506.

Pawlowski, K., Klosse, U., and De Bruijn, F. J. (1991). Characterization of a novel Azorhizobium caulinodans ORS571 two-component regulatory system, NtrY/NtrX, involved in nitrogen fixation and metabolism. Mol. Gen. Genet. 231, 124-138.

Paxman, J. J., Borg, N. A., Horne, J., Thompson, P. E., Chin, Y., Sharma, P., et al. (2009). The structure of the bacterial oxidoreductase enzyme DsbA in complex with a peptide reveals a basis for substrate specificity in the catalytic cycle of DsbA enzymes. J. Biol. Chem. 284, $17835-17845$.

Pelicic, V. (2008). Type IV pili: $e$ pluribus unum? Mol. Microbiol. 68, 827-837. 
Phillips, N. J., Steichen, C. T., Schilling, B., Post, D. M., Niles, R. K., Bair, T. B., et al. (2012). Proteomic analysis of Neisseria gonorrhoeae biofilms shows shift to anaerobic respiration and changes in nutrient transport and outermembrane proteins. PLoS ONE 7:e38303. doi: 10.1371/ journal.pone. 0038303

Piek, S., Ganguly, J., Anandan, A., Wanty, C., Stubbs, K., Scanlon, M. J., et al. (2012). "Resistance to cationic antimicrobial peptides is determined by oxidoreductases in Neisseria meningitidis," in The International Pathogenic Neisseria Conference (Wurzburg, Germany), September 09-14. Abstract 031.

Plant, L., Sundqvst, J., Zughaier, S., Lovkvist, L., Stephens, D., and Jonsson, A. (2006). Lipooligosaccharide structure contributes to multiple steps in the virulence of Neisseria meningitidis. Infect. Immun. 74, 1360-1367.

Pogliano, J., Lynch, A. S., Belin, D., Lin, E. C., and Beckwith, J. (1997). Regulation of Escherichia coli cell envelope proteins involved in protein folding and degradation by the Cpx two-component system. Genes Dev. 11, 1169-1182.

Price, N. L., and Raivio, T. L. (2009). Characterization of the Cpx regulon in Escherichia coli strain MC4100. J. Bacteriol. 191, 1798-1815.

Raetz, C. R. H., Reynolds, C. M., Trent, M. S., and Bishop, R. E. (2007). Lipid A modification systems in Gram-negative bacteria. Annu. Rev. Biochem. 76, 295-329.

Raetz, C. R. H., and Whitfield, C. (2002). Lipopolysaccharide endotoxins. Annu. Rev. Biochem. 71, 635-700.

Ram, S., Cox, A. D., Wright, J. C., Vogel, U., Getzlaff, S., Boden, R., et al. (2003). Neisserial lipooligosaccharide is a target for complement component $\mathrm{C} 4 \mathrm{~b}$. Inner core phosphoethanolamine residues define $\mathrm{C} 4 \mathrm{~b}$ linkage specificity. J. Biol. Chem. 278, 50853-50862.

Reid, E., Cole, J., and Eaves, D. J. (2001). The Escherichia coli CcmG protein fulfils a specific role in cytochrome c assembly. Biochem. J. 355, 51-58.

Ren, G., Stephan, D., Xu, Z., Zheng, Y., Tang, D., Harrison, R. S., et al. (2009). Properties of the thioredoxin fold superfamily are modulated by a single amino acid residue. J. Biol. Chem. 284, 10150-10159.

Reynolds, C. M., Kalb, S. R., Cotter, R. J., and Raetz, C. R. H. (2005). A phosphoethanolamine transferase specific for the outer 3-Deoxy-Dmanno-octulosonic acid residue of Escherichia coli lipopolysaccharide. J. Biol. Chem. 280, 21202-21211.

Rietsch, A., Belin, D., Martin, N., and Beckwith, J. (1996). An in vivo pathway for disulfide bond isomerization in Escherichia coli. Proc. Natl. Acad. Sci. U.S.A. 93, 13048-13053.

Rietsch, A., Bessette, P., Georgiou, G., and Beckwith, J. (1997). Reduction of the periplasmic disulfide bond isomerase, DsbC, occurs by passage of electrons from cytoplasmic thioredoxin. J. Bacteriol. 179, 6602-6608.

Robbins, P., Bray, D., Dankert, B., and Wright, A. (1967). Direction of chain growth in polysaccharide synthesis. Science 158, 1536-1542.

Rozhkova, A., and Glockshuber, R. (2008). Thermodynamic aspects of DsbD-mediated electron transport. J. Mol. Biol. 380, 783-788.

Rozhkova, A., Stirnimann, C. U., Frei, P., Grauschopf, U., Brunisholz, R., Grutter, M. G., et al. (2004), Structural basis and kinetics of inter- and intramolecular disulfide exchange in the redox catalyst DsbD. EMBO J. 23, 1709-1719.

Ruiz, N., Gronenberg, L. S., Kahne, D., and Silhavy, T. J. (2008). Identification of two innermembrane proteins required for the transport of lipopolysaccharide to the outer-membrane of Escherichia coli. Proc. Natl. Acad. Sci. U.S.A. 105, 5537-5542.

Ruiz, N., Kahne, D., and Silhavy, T. J. (2006). Advances in understanding bacterial outer-membrane biogenesis. Nat. Rev. Microbiol. 4, 57-66.

Rustam, T., McClean, S., Newcombe, J., McFadden, J., and Eales-Reynolds, L. J. (2006). Reduced toxicity of lipo-oligosaccharide from a phoP mutant of Neisseria meningitidis: an in vitro demonstration. J. Endotoxin. Res. 12, 39-46.

Samuel, G., and Reeves, P. (2003). Biosynthesis of $\mathrm{O}$-antigens: genes and pathways involved in nucleotide sugar precursor synthesis and O-antigen assembly. Carbohydr. Res. 338, 2503-2519.

Seib, K. L., Wu, H.-J., Srikhanta, Y. N., Edwards, J. L., Falsetta, M. L., Hamilton, A. J., et al. (2007). Characterization of the OxyR regulon of Neisseria gonorrhoeae. Mol. Microbiol. 63, 54-68.

Sinha, S., Ambur, O. H., Langford, P. R., Tonjum, T., and Kroll, J. S. (2008). Reduced DNA binding and uptake in the absence of DsbAl and DsbA2 of Neisseria meningitidis due to inefficient folding of the outer-membrane secretin PilQ. Microbiology 154, 217-225.
Sinha, S., Langford, P. R., and Kroll, J. S. (2004). Functional diversity of three different DsbA proteins from Neisseria meningitidis. Microbiology 150, 2993-3000.

Skaar, E. P., Tobiason, D. M., Quick, J. Judd, R. C., Weissbach, H., Etienne, F., et al. (2002). The outer membrane localization of the Neisseria gonorrhoeae MsrA/B is involved in survival against reactive oxygen species. Proc. Natl. Acad. Sci. U.S.A. 99, 10108-10113.

Soncini, F. C., Garcia Vescovi, E. Solomon, F., and Groisman, E. A. (1996). Molecular basis of the magnesium deprivation response in Salmonella typhimurium: identification of PhoP-regulated genes. J. Bacteriol. 178, 5092-5099.

Sperandeo, P., Cescutti, R., Villa, R., Di Benedetto, C., Candia, D., Deho, G., et al. (2007). Characterization of $l p t A$ and $l p t B$, two essential genes implicated in lipopolysaccharide transport to the outer membrane of Escherichia coli. J. Bacteriol. $189,244-253$

Sperandeo, P., Lau, F. K., Carpentieri, A., De Castro, C., Molinaro, A. Deho, G., et al. (2008). Functional analysis of the protein machinery required for transport of lipopolysaccharide to the outer membrane of Escherichia coli. J. Bacteriol. 190, 4460-4469.

Sperandeo, P., Pozzi, C., Dehò, G., and Polissi, A. (2006). Non-essential KDO biosynthesis and new essential cell envelope biogenesis genes in the Escherichia coli yrbGyhbG locus. Res. Microbiol. 157, 547-558.

Steeghs, L., Den Hartog, R., Den Boer A., Zomer, B., Roholl, P., and Van Der Ley, P. (1998). Meningitis bacterium is viable without endotoxin. Nature 392, 449-449.

Stenberg, F., Chovanec, P., Maslen, S. L., Robinson, C. V., Ilag, L. L., Von Heijne, G., et al. (2005). Protein complexes of the Escherichia col cell envelope. J. Biol. Chem. 280, 34409-34419.

Stenutz, R., Weintraub, A., and Widmalm, G. (2006). The structures of Escherichia coli Opolysaccharide antigens. FEMS Microbiol. Rev. 30, 382-403.

Stock, A., Robinson, V., and Goudreau, P. (2000). Two-component signal transduction. Annu. Rev. Biochem. $69,183-215$.

Suits, M. D., Sperandeo, P., Deho, G., Polissi, A., and Jia, Z. (2008). Novel structure of the conserved Gramnegative lipopolysaccharide transport protein $\mathrm{A}$ and mutagenesis analysis. J. Mol. Biol. 380, 476-488.
Takahashi, H., Carlson, R. W., Muszynski, A., Choudhury, B., Kim, K. S., Stephens, D. S., et al. (2008). Modification of lipooligosaccharide with phosphoethanolamine by LptA in Neisseria meningitidis enhances meningococcal adhesion to human endothelial and epithelial cells. Infect. Immun. 76, 5777-5789.

Tamayo, R., Choudhury, B., Septer, A., Merighi, M., Carlson, R., and Gunn, J. S. (2005). Identification of cptA, a PmrA-regulated locus required for phosphoethanolamine modification of the Salmonella enterica serovar Typhimurium lipopolysaccharide core. J. Bacteriol. 187, 3391-3399.

Tapley, T. L., Eichner, T., Gleiter, S., Ballou, D. P., and Bardwell, J. C. (2007). Kinetic characterization of the disulfide bond-forming enzyme DsbB. J. Biol. Chem. 282, 10263-10271.

Tefsen, B., Geurtsen, J., Beckers, F., Tommassen, J., and De Cock, H. (2005). Lipopolysaccharide transport to the bacterial outer membrane in spheroplasts. J. Biol. Chem. 280, 4504-4509.

Tettelin, H., Saunders, N., Heidelberg, J., Jeffries, A., Nelson, K., Eisen, J., et al. (2000). Complete genome sequence of Neisseria menigitidis serogroup B strain MC58. Science 287, 1809-1815.

Tinsley, C. R., Voulhoux, R., Beretti, J. L., Tommassen, J., and Nassif, X. (2004). Three homologues, including two membrane-bound proteins, of the disulfide oxidoreductase DsbA in Neisseria meningitidis: effects on bacterial growth and biogenesis of functional type IV pili. J. Biol. Chem. 279, 27078-27087.

Tokuda, H. (2009). Biogenesis of outer membranes in Gram-negative bacteria. Biosci. Biotechnol. Biochem. 73, 465-473.

Touzé, T., Tran, A. X., Hankins, J. V., Mengin-Lecreulx, D., and Trent, M. S. (2008). Periplasmic phosphorylation of lipid A is linked to the synthesis of undecaprenyl phosphate. Mol. Microbiol. 67, 264-277.

Tran, A. X., Dong, C., and Whitfield, C. (2010). Structure and functional analysis of $\mathrm{LptC}$, a conserved membrane protein involved in the lipopolysaccharide export pathway in Escherichia coli. J. Biol. Chem. 285, 33529-33539.

Tran, A. X., Trent, M. S., and Whitfield, C. (2008). The LptA protein of Escherichia coli is a periplasmic lipid A-binding protein involved in the lipopolysaccharide export pathway. J. Biol. Chem. 283, 20342-20349. 
Trent, M. S., and Raetz, C. R. H. (2002). Cloning of EptA, the lipid A phosphoethanolamine transferase associated with polymyxin resistance. J. Endotoxin. Res. 8, 158

Trent, M., Ribeiro, A., Doerrler, W., Lin, S., Cotter, R., and Raetz, C. (2001a). Accumulation of a polyisoprenelinked amino sugar in polymyxinresistant Salmonella typhimurium and Escherichia coli. J. Biol. Chem. 276, 43132-43144.

Trent, M. S., Pabich, W., Raetz, C. R. H., and Miller, S. I. (2001b). A PhoP/PhoQ-induced lipase (PagL) that catalyzes 3-O-deacylation of lipid A precursors in membranes of Salmonella typhimurium. J. Biol. Chem. 276, 9083-9092.

Trent, M. S., Ribeiro, A. A., Lin, S., Cotter, R. J., and Raetz, C. R. (2001c). An inner membrane enzyme in Salmonella and Escherichia coli that transfers 4amino-4-deoxy-L-arabinose to lipid A: induction on polymyxinresistant mutants and role of a novel lipid-linked donor. J. Biol. Chem. 276, 43122-43131.

Trent, M. S., Stead, C. M., Tran, A. X., and Hankins, J. V. (2006). Diversity of endotoxin and its impact on pathogenesis. J. Endotoxin. Res. 12, 205-223.

Tsai, C. M., Mocca, L. F., and Frasch, C. E. (1987). Immunotype epitopes of Neisseria meningitidis lipooligosaccharide types 1 through 8 . Infect. Immun. 55, 1652-1656.

Tzeng, Y., Ambrose, K., Zughaier, S., Zhou, X., Miller, Y., Shafer, W., et al. (2005). Cationic antimicrobial peptide resistance in Neisseria meningitidis. J. Bacteriol. 187, 53587-55396.

Tzeng, Y. L., Datta, A., Ambrose, K., Lo, M., Davies, J. K., Carlson, R. W., et al. (2004). The MisR/MisS two-component regulatory system influences inner core structure and immunotype of lipooligosaccharide in Neisseria meningitidis. J. Biol. Chem. 279, 35053-35062.

Tzeng, Y. L., Kahler, C. M., Zhang, X., and Stephens, D. S. (2008). MisR/MisS two-component regulon in Neisseria meningitidis. Infect. Immun. 76, 704-716.

Tzeng, Y. L., Zhou, X., Bao, S., Zhao, S., Noble, C., and Stephens, D. S. (2006). Autoregulation of the MisR/MisS two-component signal transduction system in Neisseria meningitidis. J. Bacteriol. 188, 5055-5065.

Vertommen, D., Ruiz, N., Leverrier, P., Silhavy, T. J., and Collet, J.F. (2009). Characterization of the role of the Escherichia coli periplasmic chaperone SurA using differential proteomics. Proteomics 9, 2432-2443.

Vinogradov, E. V., Van Der Drift, K., Thomas-Oates, J. E., Meshkov, S., Brade, H., and Holst, O. (1999). The structures of the carbohydrate backbones of the lipopolysaccharides from Escherichia coli rough mutants F470 (R1 core type) and F576 (R2 core type). Eur. J. Biochem. 261, 629-639.

Virji, M., Makepeace, K., Peak, I. R., Ferguson, D. J., Jennings, M. P., and Moxon, E. R. (1995). Opcand pilus-dependent interactions of meningococci with human endothelial cells: molecular mechanisms and modulation by surface polysaccharides. Mol. Microbiol. 18, 741-754.

Vivian, J. P., Scoullar, J., Rimmer, K., Bushell, S. R., Beddoe, T., Wilce, M. C. J., et al. (2009). Structure and function of the oxidoreductase DsbA1 from Neisseria meningitidis. J. Mol. Biol. 394, 931-943.

Vivian, J. P., Scoullar, J., Robertson, A. L., Bottomley, S. P., Horne, J., Chin, Y., et al. (2008). Structural and biochemical characterization of the oxidoreductase NmDsbA3 from Neisseria meningitidis. J. Biol. Chem. 283, 32452-32461.

Vogt, S. L., Nevesinjac, A. Z., Humphries, R. M., Donnenberg, M. S., Armstrong, G. D., and Raivio, T. L. (2010). The Cpx envelope stress response both facilitates and inhibits elaboration of the enteropathogenic Escherichio coli bundle-forming pilus. Mol. Microbiol. 76, 1095-1110.

Vogt, S. L., and Raivio, T. L. (2012). Just scratching the surface: an expanding view of the Cpx envelope stress response. FEMS Microbiol. Lett. 326, 2-11.

Volokhina, E. B., Grijpstra, J., Stork, M., Schilders, I., Tommassen, J. and Bos, M. P. (2011). Role of the periplasmic chaperones Skp, SurA, and DegQ in outer membrane protein biogenesis in Neisseria meningitidis. J. Bacteriol. 193, 1612-1621.

Wakarchuk, W., Martin, A., Jennings, M. P., Moxon, E. R., and Richards, J. C. (1996). Functional relationships of the genetic locus encoding the glycosyltransferase enzymes involved in expression of the lacto-N-neotetraose terminal lipopolysaccharide structure in Neisseria meningitidis. J. Biol. Chem. 271, 19166-19173.

Wakarchuk, W. W., Gilbert, M., Martin, A., Wu, Y., Brisson, J. R., Thibault, P., et al. (1998). Structure of an alpha-2, 6-sialylated lipooligosaccharide from Neisseria meningitidis immunotype L1. Eur. J. Biochem. $254,626-633$.

Walker, K. W., and Gilbert, H. F (1997). Scanning and escape during protein-disulfide isomerase-assisted protein folding. J. Biol. Chem. 272, 8845-8848.

Waters, L. S., and Storz, G. (2009). Regulatory RNAs in bacteria. Cell 136, 615-628

Whitehead, R. N., and Cole, J. A. (2006). Different responses to nitrate and nitrite by the model organism Escherichia coli and the human pathogen Neisseria gonorrhoeae. Biochem. Soc. Trans. 34, 111-114.

Whitfield, C. (1995). Biosynthesis of lipopolysaccharide $\mathrm{O}$ antigens. Trends Microbiol. 3, 178-185.

Whitfield, C., Amor, P. A., and KöPlin, R. (1997). Modulation of the surface architecture of Gram-negative bacteria by the action of surface polymer:lipid A-core ligase and by determinants of polymer chain length. Mol. Microbiol. 23, 629-638.

Wright, J. C., Hood, D. W., Randle, G. A., Makepeace, K., Cox, A. D. $\mathrm{Li}$, J., et al. (2004). lpt6, a gene required for addition of phosphoethanolamine to inner-core lipopolysaccharide of Neisseria meningitidis and Haemophilus influenzae. J. Bacteriol. 186, 6970-6982.

Wu, T., McCandlish, A. C. Gronenberg, L. S., Chng, S. S., Silhavy, T. J., and Kahne, D. (2006) Identification of a protein complex that assembles lipopolysaccharide in the outer membrane of Escherichia coli. Proc. Natl. Acad. Sci. U.S.A. 103, 11754-11759.

Wulfing, C., and Rappuoli, R. (1997). Efficient production of heat-labile enterotoxin mutant proteins by overexpression of $d s b A$ in a $\operatorname{deg} P$ deficient Escherichia coli strain. Arch. Microbiol. 167, 280-283.

Wunderlich, M., Jaenicke, R., and Glockshuber, R. (1993). The redox properties of protein disulfide isomerase (DsbA) of Escherichia col result from a tense conformation of its oxidised form. J. Mol. Biol. 233, 559-566.

Yamamoto, K., Hirao, K., Oshima, T., Aiba, H., Utsumi, R., and Ishihama A. (2005). Functional characterization in vitro of all two-component signal transduction systems from Escherichia coli. J. Biol. Chem. 280, 1448-1456.

Yethon, J. A., Gunn, J. S., Ernst, R. K., Miller, S. I., Laroche, L., Malo, D., et al. (2000a). Salmonella enterica serovar Typhimurium waaP mutants show increased susceptibility to polymyxin and loss of virulence in vivo. Infect. Immun. 68, 4485-4491.

Yethon, J. A., Vinogradov, E., Perry, M. B., and Whitfield, C. (2000b) Mutation of the lipopolysaccharide core glycosyltransferase encoded by waaG destabilizes the outer membrane of Escherichia coli by interfering with core phosphorylation. J. Bacteriol. 182, 5620-5623.

Yethon, J. A., Heinrichs, D. E., Monteiro, M. A., Perry, M. B., and Whitfield, C. (1998). Involvement of waaY, waaQ, and waaP in the modification of Escherichia coli lipopolysaccharide and their role in the formation of a stable outer membrane. J. Biol. Chem. 273, 26310-26316

Yethon, J. A., and Whitfield, C. (2001). Purification and characterization of WaaP from Escherichia coli, a lipopolysaccharide kinase essential for outer membrane stability. J. Biol. Chem. 276, 5498-5504.

Yu, J., and Kroll, J. S. (1999). DsbA: a protein-folding catalyst contributing to bacterial virulence. Microbes Infect. 1, 1221-1228.

Yu, J., Webb, H., and Hirst, T. (1992). A homologue of the Escherichia coli DsbA protein involved in disulfide bond formation is required for enterotoxin biogenesis in Vibrio cholerae. Mol. Microbiol. 6, 1949-1958.

Zamyatina, A., Gronow, S., Oertelt, C., Puchberger, M., Brade, H., and Kosma, P. (2000). Efficient chemical synthesis of the two anomers of ADP-L-glycero- and D-glycero-D-manno-heptopyranose allows the determination of the substrate specificities of bacterial heptosyltransferases. Angew. Chem. Int. Ed. Engl. 39, 4150-4153.

Zapun, A., Missiakas, D., Raina, S., and Creighton, T. E. (1995). Structural and functional characterization of DsbC, a protein involved in disulfide bond formation in Escherichia coli. Biochemistry 34, 5075-5089.

Zasloff, M. (2002). Antimicrobial peptides of multicellular organisms. Nature 415, 389-395.

Zhang, H., and Donnenberg, M. (1996). DsbA is required for stability of the type IV pilin of enteropathogenic Escherichia coli. Mol. Microbiol. 21, 787-797.

Zhao, S., Montanez, G. E., Kumar, P., Sannigrahi, S., and Tzeng, Y. L. (2010). Regulatory role of the MisR/S two-component system in hemoglobin utilization in Neisseria 
meningitidis. Infect. Immun. 78, 1109-1122.

Zhou, Y., Cierpicki, T., Jimenez, R. H., Lukasik, S. M., Ellena, J. F., Cafiso, D. S., et al. (2008). NMR solution structure of the integral membrane enzyme DsbB: functional insights into DsbB-catalyzed disulfide bond formation. Mol. Cell 31, 896-908.

Zhou, Z., Lin, S., Cotter, R. J., and Raetz, C. R. H. (1999). Lipid A modifications characteristic of Salmonella typhimurium are induced by $\mathrm{NH} 4 \mathrm{VO} 3$ in Escherichia coli K12. J. Biol. Chem. 274, 18503-18514.

Zhou, Z., Ribeiro, A. A., Lin, S., Cotter, R. J., Miller, S. I., and Raetz, C.
R. H. (2001). Lipid A modifications in polymyxin-resistant Salmonella typhimurium. J. Biol. Chem. 276, 43111-43121.

Zhou, Z., Ribeiro, A. A., and Raetz, C. R. H. (2000). Highresolution NMR spectroscopy of lipid A molecules containing 4-amino-4-deoxy-l-arabinose and phosphoethanolamine substituents. J. Biol. Chem. 275, 13542-13551.

Zhou, Z., White, K. A., Polissi, A., Georgopoulos, C., and Raetz, C. R. (1998). Function of Escherichia coli MsbA, an essential ABC family transporter, in lipid A and phospholipid biosynthesis. J. Biol. Chem. 273, 12466-12475.
Zhu, P., Klutch, M. J., Bash, M. C., Tsang, R. S., Ng, L. K., and Tsai, C. M. (2002). Genetic diversity of three lgt loci for biosynthesis of lipooligosaccharide (LOS) in Neisseria species. Microbiology 148, 1833-1844.

Conflict of Interest Statement: The authors declare that the research was conducted in the absence of any commercial or financial relationships that could be construed as a potential conflict of interest.

Received: 04 September 2012; paper pending published: 10 October 2012; accepted: 01 December 2012; published online: 20 December 2012.

Citation: Piek S and Kahler CM (2012) A comparison of the endotoxin biosynthesis and protein oxidation pathways in the biogenesis of the outer membrane of Escherichia coli and Neisseria meningitidis. Front. Cell. Inf. Microbio. 2:162. doi: 10.3389/fcimb.2012.00162

Copyright (c) 2012 Piek and Kahler. This is an open-access article distributed under the terms of the Creative Commons Attribution License, which permits use, distribution and reproduction in other forums, provided the original authors and source are credited and subject to any copyright notices concerning any third-party graphics etc. 